\title{
A FAST ALGORITHM FOR GAUSSIAN TYPE QUADRATURE FORMULAE WITH MIXED BOUNDARY CONDITIONS AND SOME LUMPED MASS SPECTRAL APPROXIMATIONS
}

\author{
ABDELKRIM EZZIRANI AND ALLAL GUESSAB
}

\begin{abstract}
After studying Gaussian type quadrature formulae with mixed boundary conditions, we suggest a fast algorithm for computing their nodes and weights. It is shown that the latter are computed in the same manner as in the theory of the classical Gauss quadrature formulae. In fact, all nodes and weights are again computed as eigenvalues and eigenvectors of a real symmetric tridiagonal matrix. Hence, we can adapt existing procedures for generating such quadrature formulae. Comparative results with various methods now in use are given.

In the second part of this paper, new algorithms for spectral approximations for second-order elliptic problems are derived. The key to the efficiency of our algorithms is to find an appropriate spectral approximation by using the most accurate quadrature formula, which takes the boundary conditions into account in such a way that the resulting discrete system has a diagonal mass matrix. Hence, our algorithms can be used to introduce explicit resolutions for the time-dependent problems. This is the so-called lumped mass method. The performance of the approach is illustrated with several numerical examples in one and two space dimensions.
\end{abstract}

\section{INTRODUCTION AND MOTIVATIONS}

Consider a fixed nonnegative measure $d \sigma$ with support in the interval $[-1,1]$. The standard approach to estimating integrals of the form

$$
I_{\sigma}(f):=\int_{-1}^{1} f(x) d \sigma
$$

is to seek quadrature formulae

$$
G_{n, m}(f):=\sum_{i=1}^{n} \alpha_{i, n} f\left(x_{i, n}\right)+\sum_{j=1}^{m} \sum_{l=0}^{2 k_{j}+1} \beta_{j, l, n} f^{(l)}\left(a_{j}\right),
$$

where $a_{1}<a_{2}<\ldots<a_{m}$ are given real numbers. The free nodes $\left\{x_{i, n}\right\}$ and the weights $\left\{\alpha_{i, n}\right\}$ and $\left\{\beta_{j, l, n}\right\}$ are commonly chosen such that $G_{n, m}$ has maximum

Received by the editor May 6, 1997.

1991 Mathematics Subject Classification. Primary 65D30, 65D32, 65N35.

Key words and phrases. Quadrature formulae, Gaussian quadrature formulae, spectral methods, lumped mass methods, quasi-orthogonal polynomials, algorithms.

The work of the second author was supported by the CNRS grant INTAS-94-4070.

(C)1999 American Mathematical Society 
degree of exactness (MDE); that is,

$$
G_{n, m}(f)=\int_{-1}^{1} f(x) d \sigma, \quad \forall f \in \mathcal{P}_{s},
$$

where $s=\sup \left\{k: R_{n, m}\left(\mathcal{P}_{k}\right)=0\right\}$, with $R_{n, m}(f):=\int_{-1}^{1} f(x) d \sigma-G_{n, m}(f)$ and $\mathcal{P}_{k}$ denotes the linear space of polynomials on $[-1,1]$ of total degree at most $k$.

In a recent paper, Guessab and Milovanović [23] have presented a new method for numerical construction of the generalized Gaussian type quadrature formula

$$
\int_{-1}^{1} f(t) d \sigma=A_{n} f^{\prime}(-1)+B_{n} f^{\prime}(1)+\sum_{i=1}^{n} \lambda_{i, n} f\left(x_{i, n}\right)+R_{n}(f)
$$

which has $\mathrm{MDE}=2 n+1$. The key to this algorithm is to determine numerically the coefficients that appear in the three-term recurrence relation, satisfied by certain quasi-orthogonal polynomials $\left\{\pi_{n}\left(., d \sigma\left(. ; \pi_{n}\right)\right)\right\}_{n=0,1,2, \ldots}$ implicitly defined. A notable disadvantage of this construction is that the method requires the solution of a nonlinear system which is, unfortunately, very ill-conditioned if $n$ is moderately large.

In this paper, we propose to compute the nodes $x_{i, n}$ and the weights $\lambda_{i, n}$ and $\omega_{j, n}$ (provided they exist) of the more general quadrature formulae

$$
\begin{aligned}
\int_{-1}^{1} f(t) d \sigma & =Q_{n, k}(f)+R_{n, k}(f) \\
& =\sum_{i=1}^{n} \lambda_{i, n} f\left(x_{i, n}\right)+\sum_{j=1}^{k} \omega_{j, n} C_{j}(f)+R_{n, k}(f),
\end{aligned}
$$

where $C_{j}, j=1, \ldots, k$, are given linear functionals of the form

$$
C_{l}(f):=\sum_{m=0}^{q_{l}-1} a_{l m} f^{(m)}(-1)+\sum_{p=0}^{q_{l}^{\prime}-1} b_{l p} f^{(p)}(1), \quad l=1, \ldots, k,
$$

that has $\mathrm{MDE}=2 n+k-1$. We call these quadrature formulae generalized GaussLobatto-Birkhoff quadrature formulae. This definition is justified by the analogy between some well-known quadrature formulae and (2). Indeed, note that $\operatorname{MDE}\left(Q_{n, k}\right)$ is equal to the number of "free" parameters appearing on $Q_{n, k}$, and that, as special cases, we obtain the well-known Gauss quadrature formula, as well as the quadrature formulae usually associated with the names of Radau, Lobatto, Birkhoff and the quadrature formula (1). Here, we propose a numerical scheme for the construction of such Gaussian quadrature formulae. The main advantage of the new algorithm is to extend the well-known method of Golub and Welsch [19] for ordinary Gaussian quadrature formulae to (2). We shall in fact show, under additional assumptions on the functionals $C_{l}$ which guarantee existence of $Q_{n, k}$, that all nodes and weights can again be (and have been) computed as eigenvalues and eigenvectors of a specific real symmetric tridiagonal matrix. Hence, an efficient and stable numerical method for the construction of a new class of quadrature formulae is almost immediate. Also, our approach is conceptually simpler than the method given in [23] and leads to considerable savings in computational time. Moreover, it will be shown that (2) possesses most of the desirable properties of the generalized Gauss-Lobatto quadrature formulae, and we obtain, for some boundary functionals $C_{l}$, an explicit expression for the "boundary" weights $\omega_{j, n}$. Comparative numerical results with various existing quadrature formulae are given. Judging by our numerical examples, the new quadrature formulae appear to be very effective. 
One of the main contributions of this work is to derive, in particular, a new approach for developing efficient algorithms for spectral approximations well suited for second-order elliptic problems. The advantages of this approach are that the discretization schemes are constructed with a judicious choice of an appropriate quadrature formula of a suitable MDE, which takes the boundary conditions into account in such a way that the resulting discrete systems have automatically diagonal mass matrices. This property is important in the sense of required computing time, because this method can be used to introduce explicit schemes for the timedependent problems. This is the so-called lumped mass method. Of course mass lumping is not a new concept (cf. for example [24], [37], among others) but our paper marks the first use of the method to obtain spectral approximations for approximating the second-order elliptic problems with various boundary conditions common in applications. The mass lumping has only been obtained for Helmholtz equation with Dirichlet boundary conditions; this case is particularly easy, since we can use as collocation points the nodes of the classical Gauss-Lobatto quadrature formula (cf. [37]). The approach can be applied to more general problems. We explain briefly how to efficiently solve the biharmonic equation with mass lumping. It provides an initial motivation for this investigation. Some numerical experiments are presented: the result shows that the new set of nodes gives better results compared to the standard Gauss-Lobatto nodes commonly used.

The remainder of this paper is organized as follows. In the next section, we state and prove, under certain assumptions about boundary conditions, existence and uniqueness of (2). We also develop some of their properties. The main results given in $\S 3$ show how such quadrature formulae can be constructed, and in $\S 4$, examples of such quadrature formulae are given. We also compare (2) and the generalized Gauss-Lobatto quadrature formulae. Section 5 presents a number of applications to lumped mass spectral approximations for second-order elliptic problems with various boundary conditions. Illustrative examples, in one and two space dimensions, are included to show the accuracy of the proposed methods compared with existing methods, when they are applied on a standard set of test problems. Some concluding remarks are given in the final section. We end this paper with an appendix containing several tables of nodes and weights of (2).

\section{Existence of THE QUADRATURES AND SOME OF THEIR PROPERTIES}

In this section, under additional assumptions on the functionals $C_{l}$, we show that there exists one and only one quadrature formula of type (2). The existence results, that are valid for a more general class of quadrature formulae, have been obtained by Micchelli and Pinkus [30, Theorem 3.1]. The main difference between our proof and that in [30] (cf., in particular $\S 3$ of [30]) is that our method uses the classical Krein's theorem [27]. We also show that (2) possesses most of the desirable properties of the classical Gaussian quadrature formulae; for example the free nodes are all in the support of the measure, the weights $\lambda_{i, n}$ are all positive, and the interior nodes have the interlacing property and are a solution of an extremal problem.

We first introduce some basis notations that will be used in the subsequent sections. Let the function $f$ in $(2)$ be differentiable on $[-1,1]$ as many times as 
needed. For given linear functionals

$$
C_{l}(f)=\sum_{m=0}^{q_{l}-1} a_{l m} f^{(m)}(-1)+\sum_{p=0}^{q_{l}^{\prime}-1} b_{l p} f^{(p)}(1), \quad l=1, \ldots, k,
$$

we shall denote by $S_{N-1, k}$ the space defined by

$$
S_{N-1, k}=\left\{P \in \mathcal{P}_{N+k-1}, C_{l}(P)=0, l=1, \ldots, k\right\} .
$$

Throughout this paper, we assume that for every integer $N$ the set of the linear functionals $\left\{C_{l}\right\}_{l=1}^{k}$ is independent over $\mathcal{P}_{N+k-1}$, that is,

$$
\operatorname{rank}\left\|C_{i}\left(x^{j}\right)\right\|_{i=1, j=0}^{k, N+k-1}=k,
$$

and

$$
\begin{aligned}
& S_{N-1, k} \text { has a basis of } N \text { functions, } \\
& \text { that form a Chebyshev system on }[-1,1] .
\end{aligned}
$$

These standing hypotheses will not be mentioned explicitly in the results of this paper.

Recall that a linear subspace $\mathcal{M}_{m}$ of $C[-1,1]$ of dimension $m+1$ spanned by the functions $u_{0}(t), \ldots, u_{m}(t)$ is called a Chebyshev system on $[-1,1]$ if and only if for any points $-1 \leq x_{0}<\ldots<x_{m} \leq 1$ and any data $y_{0}, y_{1}, \ldots, y_{m}$, there exists a unique $u \in \mathcal{M}_{m}$ satisfying

$$
u\left(x_{i}\right)=y_{i}, \quad i=0,1, \ldots, m .
$$

In particular, it follows that any nontrivial $u \in \mathcal{M}_{m}$ has at most $m$ distinct zeros.

In order to illustrate assumptions (4) and (5), we list below a few known functionals that are independent and for which $S_{N-1, k}$ forms a Chebyshev system on $[-1,1]$ with dimension $N$. These functionals have been studied in detail in [30, p. $216]$.

Example 2.1. Functionals with Hermite boundary conditions

$$
\begin{array}{ll}
C_{i}(f)=f^{(i)}(-1), \quad i=1, \ldots, p, \\
C_{j}(f)=f^{(j)}(1), \quad j=p+1, \ldots, k .
\end{array}
$$

Functionals with anti-symmetric boundary conditions

$$
C_{i}(f)=f^{(i)}(-1)+f^{(i)}(1), \quad i=0,1, \ldots, k-1 .
$$

Functionals with Birkhoff boundary conditions

$$
\begin{aligned}
& C_{\mu}(f)=f^{\left(i_{\mu}\right)}(-1), \quad \mu=1, \ldots, p, \\
& C_{\mu}(f)=f^{\left(j_{\mu}\right)}(1), \quad \mu=p+1, \ldots, k,
\end{aligned}
$$

where $0 \leq i_{1} \leq \ldots \leq i_{p} \leq N+k-1,0 \leq j_{1} \leq \ldots \leq j_{p} \leq N+k-1$, and $M_{\nu-1}^{\prime}+N \geq \nu, \nu=N+1, \ldots, N+k$, where $M_{\nu}^{\prime}$ counts the number of integers in $\left\{i_{1}, \ldots, i_{p}, j_{1}, \ldots, j_{q}\right\}$ less than or equal to $\nu$.

Another important class of functionals, which satisfy certain determinantal conditions (see [30, p. 216, Example 3.5]), is the set of functionals with separate boundary conditions

$$
\begin{array}{ll}
C_{l}(f)=\sum_{j=0}^{q_{l}-1} a_{l j} f^{(j)}(-1), & l=1, \ldots, p, \\
C_{l}(f)=\sum_{j=0}^{q_{l}^{\prime}-1} b_{l j} f^{(j)}(1), & l=p+1, \ldots, k .
\end{array}
$$

Also, for the general case (3) examples are given in [30, p. 214]. 
Note that if the functionals (3) are of the form (6), then the quadrature formulae (2) are reduced to the so-called generalized Lobatto quadrature formulae, whose existence and uniqueness have been proved by Stancu [40]. A numerical algorithm for their construction was given by Golub and Kautsky in [21]. Another algorithm has also been developed recently by Bernardi and Maday [3], who presented some useful information about the boundary weights. Also, for the functionals with Birkhoff type boundary conditions (7), we obtain Micchelli and Rivlin type quadrature formulae [31]. Existence and uniqueness of such quadrature formulae was proved in [31], but the problem of their construction is still open. The most difficult part is investigating the zeros of the polynomials that provide the nodes. Our main achievement in the following theorem is in the manner of establishing the existence, the uniqueness, and some properties of (2).

Theorem 2.1. There exists a unique quadrature formula of type (2), which integrates exactly all polynomials of degree at most $2 n+k-1$. The nodes $x_{1, n}, \ldots, x_{n, n}$ are all in the open interval $(-1,1)$, and their weights $\lambda_{1, n}, \ldots, \lambda_{n, n}$ are all positive.

Proof. The result is an immediate consequence of the Krein theorem [27], which states that there exists a unique quadrature formula of the form

$$
\int_{-1}^{1} f(t) d \sigma=\sum_{i=1}^{n} \lambda_{i, n}^{K} f\left(x_{i, n}^{K}\right)+R_{n}^{K}(f)
$$

which integrates all polynomials of $\mathcal{S}_{2 n-1, k}$. Moreover,

$$
x_{i, n}^{K} \in(-1,1) \text { and } \lambda_{i, n}^{K}>0, \quad i=1, \ldots, n .
$$

With the help of this result, the proof becomes very easy and we prefer to present it here to keep the paper self-contained. We are now ready to prove the uniqueness of (2).

I) Uniqueness result. Suppose that there is another quadrature formula of the form

$$
\int_{-1}^{1} f(t) d \sigma=\sum_{i=1}^{n} \bar{\lambda}_{i, n} f\left(\bar{x}_{i, n}\right)+\sum_{j=1}^{k} \bar{\omega}_{j, n} C_{j}(f)+\bar{R}_{n, k}(f),
$$

having the desired property, and which is exact also for all polynomials from $\mathcal{P}_{2 n+k-1}$. Suppose that $x_{i, n} \neq \bar{x}_{i, n}$ for some $i$, and define the polynomial $Q_{i} \in$ $\mathcal{P}_{2 n+k-1}$ such that

$$
\begin{aligned}
& Q_{i}\left(x_{l, n}\right)=0, \quad l=1, \ldots, n, \\
& C_{j}\left(Q_{i}\right)=0, \quad j=1, \ldots, k, \\
& Q_{i}\left(\bar{x}_{j, n}\right)=0, \quad j=1, \ldots, n, \quad j \neq i, \\
& Q_{i}\left(\bar{x}_{i, n}\right)=1 .
\end{aligned}
$$

The existence of $Q_{i}$ follows from the fact that $\mathcal{S}_{2 n-1, k}$ is a Chebyshev system of dimension $2 n$. Then,

$$
\int_{-1}^{1} Q_{i}(t) d \sigma=0
$$

by the first quadrature formula, and

$$
\int_{-1}^{1} Q_{i}(t) d \sigma=\bar{\lambda}_{i, n}>0
$$


by the second. This leads to a contradiction, so that $x_{i, n}=\bar{x}_{i, n}, i=1, \ldots, n$. It follows easily that $\lambda_{i, n}=\bar{\lambda}_{i, n}, i=1, \ldots, n$, since $\mathcal{S}_{2 n-1, k}$ is a Chebyshev system of dimension $2 n$.

The equality of quadrature weights $\omega_{j, n}=\bar{\omega}_{j, n}, j=1, \ldots, k$, is an immediate consequence of the rank property (4).

II) Existence result. We require of a good quadrature formula that its nodes be in the support of the measure. We show that, for (2), this holds true. To this end, let $f$ be a given function on $[-1,1]$ and let $\mathcal{I}_{2 n+k-1}(f ;$.$) be the (2 n+k-1)$-th degree Lagrange interpolating polynomial based on the data

$$
\left\{f\left(x_{i, n}^{K}\right), \quad i=1, \ldots, n ; f\left(x_{i, n}\right), \quad i=n+1, \ldots, 2 n ; C_{j}(f), \quad j=1, \ldots, k\right\},
$$

where $x_{i, n}^{K}, i=1, \ldots, n$, are the nodes of the Krein quadrature formula (9), and $x_{i, n}, i=n+1, \ldots, 2 n$, are $n$ distinct points on the interval $(-1,1)$ such that $x_{j, n} \neq$ $x_{i, n}^{K}$ for all $j=n+1, \ldots, 2 n$ and $i=1, \ldots, n$. Then, it is well-known that

$$
\mathcal{I}_{2 n+k-1}(f ; t)=\sum_{i=1}^{n} f\left(x_{i, n}^{K}\right) h_{i, n}(t)+\sum_{i=n+1}^{2 n} f\left(x_{i, n}\right) h_{i, n}(t)+\sum_{j=1}^{k} C_{j}(f) l_{j, n}(t),
$$

where $h_{i, n}$ and $l_{j, n}$ are the so-called fundamental functions (the existence and uniqueness of $h_{i, n}$, and $l_{j, n}$ follow from the fact that $\mathcal{S}_{2 n-1, k}$ is a Chebyshev system of dimension $2 n$ and the rank property (4)). Since $\mathcal{I}_{2 n+k-1}(f ;)=$.$f for all$ $f \in \mathcal{P}_{2 n+k-1}$, an integration of $\mathcal{I}_{2 n+k-1}(f ; t)$ leads to the following quadrature formula:

$$
\int_{-1}^{1} f(t) d \sigma=\sum_{i=1}^{n} \lambda_{i, n} f\left(x_{i, n}^{K}\right)+\sum_{i=n+1}^{2 n} \lambda_{i, n} f\left(x_{i, n}\right)+\sum_{j=1}^{k} \omega_{j, n} C_{j}(f)+R_{n, k}(f),
$$

where

$$
\lambda_{i, n}=\int_{-1}^{1} h_{i, n}(t) d \sigma \text { and } \omega_{j, n}=\int_{-1}^{1} l_{j, n}(t) d \sigma .
$$

Since $h_{i, n} \in \mathcal{S}_{2 n-1, k}$, for all $i=n+1, \ldots, 2 n$, and vanishes at the nodes of (9), we obtain $\lambda_{i, n}=0, i=n+1, \ldots, 2 n$. We have also $\lambda_{i, n}=\lambda_{i, n}^{K}, i=1, \ldots, n$, this fact following immediately by using the uniqueness of (9). Therefore, (10) is a quadrature formula of the form (2). The proof is complete.

The proof shows that the interior nodes of (2) are those of the Krein quadrature formula (9).

The next theorem says that, as in the classical Gaussian quadrature formulae, the interior nodes of (2) have the interlacing property.

Theorem 2.2. Let

$$
\int_{-1}^{1} f(t) d \sigma=\sum_{i=1}^{n} \lambda_{i, n} f\left(x_{i, n}\right)+\sum_{j=1}^{k} \omega_{j, n} C_{j}(f)+R_{n, k}(f), R_{n, k}\left(\mathcal{P}_{2 n+k-1}\right)=0
$$


and

$$
\begin{aligned}
\int_{-1}^{1} f(t) d \sigma=\sum_{i=1}^{n+1} \lambda_{i, n+1} f\left(x_{i, n+1}\right)+\sum_{j=1}^{k} \omega_{j, n+1} C_{j}(f)+R_{n+1, k}(f) \\
R_{n+1, k}\left(\mathcal{P}_{2 n+k+1}\right)=0
\end{aligned}
$$

with $-1<x_{1, n}<\ldots<x_{n, n}<1$ and $-1<x_{1, n+1}<\ldots<x_{n+1, n+1}<1$. Then the following interlacing property holds:

$$
-1<x_{1, n+1}<x_{1, n}<x_{2, n+1}<x_{2, n}<\ldots<x_{n, n}<x_{n+1, n+1}<1 .
$$

Proof. Let us assume on the contrary that there exists $\mu$ with

$$
x_{\mu, n} \notin\left[x_{\mu, n+1}, x_{\mu+1, n+1}\right] .
$$

Define the polynomial $Q_{\mu} \in \mathcal{P}_{2 n+k-1}$ by the interpolation conditions

$$
\begin{aligned}
& Q_{\mu}\left(x_{i, n}\right)=0, \quad i=1, \ldots, n, \\
& C_{j}\left(Q_{\mu}\right)=0, \quad j=1, \ldots, k, \\
& Q_{\mu}\left(x_{j, n+1}\right)=0, \quad j=1, \ldots, n+1, j \neq \mu \text { and } j \neq \mu+1, \\
& Q_{\mu}\left(x_{\mu, n+1}\right)=1 .
\end{aligned}
$$

Since $\mathcal{S}_{2 n-1, k}$ is a Chebyshev system of dimension $2 n$ and the number of the zero evaluations of $Q_{\mu}$ is maximal, we have $Q_{\mu}\left(x_{\mu+1, n+1}\right)>0$. Then,

$$
\int_{-1}^{1} Q_{\mu}(t) d \sigma=0
$$

by the first quadrature formula, and

$$
\int_{-1}^{1} Q_{\mu}(t) d \sigma=\lambda_{\mu, n+1} Q_{\mu}\left(x_{\mu, n+1}\right)+\lambda_{\mu+1, n+1} Q_{\mu}\left(x_{\mu+1, n+1}\right)>0
$$

by the second. This leads to a contradiction, and the interlacing property is proved.

We conclude this section with a remark about an extremal property of (2). We recall first of all that the ordinary Gauss quadrature formula [16] has the remarkable property that its nodes minimize the integral

$$
\left|\int_{-1}^{1} \prod_{i=1}^{n}\left(t-x_{i, n}\right)^{2} d \sigma\right|
$$

over the $n$-simplex $-1 \leq x_{1, n} \leq \ldots \leq x_{n, n} \leq 1$. Let $\Omega_{2 n+k}$ be the unique polynomial belonging to $\mathcal{P}_{2 n+k}$ with the leading coefficient 1 and satisfying the interpolation conditions

$$
\begin{aligned}
\Omega_{2 n+k}\left(x_{i, n}\right) & =\Omega_{2 n+k}^{\prime}\left(x_{i, n}\right)=0, & & i=1, \ldots, n, \\
C_{j}\left(\Omega_{2 n+k}\right) & =0, & & j=1, \ldots, k .
\end{aligned}
$$

The relation between the extremal problem,

$$
\left|\int_{-1}^{1} \Omega_{2 n+k}(t) d \sigma\right| \rightarrow \text { minimum }
$$

and the interior nodes of (2) was given by Micchelli and Rivlin [31] in the case of boundary conditions of Birkhoff type (7). 
Note that (11) is the major term in the estimate of the error

$$
R_{n, k}(f)=\int_{-1}^{1} f(t) d \sigma-Q_{n, k}(f) .
$$

Indeed, if $f$ is $n+2 k$ times continuously differentiable, then, as in the classical Hermitian case, we can easily show for (7) (see [28, Theorem 7.5]) that the modulus of the error has the representation

$$
\left|R_{n, k}(f)\right| \leq M\left|\int_{-1}^{1} \Omega_{2 n+k}(t) d \sigma\right|,
$$

with $M=\max _{-1 \leq t \leq 1}\left|f^{(2 n+k)}(t)\right| /((2 n+k) !)$.

The extremal property (11) has been extended to Birkhoff type nodes; see, for example, the papers by Jetter [25], Bojanov and Nikolov [6]. We also mention that this property has been examined in the case of a wide class of functions including smooth functions (not necessarily polynomials). See Bojanov, Braess and Dyn [5], Bojanov, Grozev and Zhensykbaev [7].

\section{QUaSI-ORTHOGONAL POLYNOMIALS AND JACOBI MATRICES}

In this section, we show that the interior nodes of (2) are zeros of certain quasiorthogonal polynomials. Then, we establish that the quasi-orthogonal polynomials that lead to (2) can be represented as characteristic polynomials of symmetric tridiagonal matrices. Because of such a representation, the nodes and weights of (2) can be computed in a stable and efficient way.

Let $d \sigma$ be a nonnegative measure with support in the interval $[-1,1]$, and let $\left\{\pi_{n}(.)=\pi_{n}(. ; d \sigma)\right\}_{n=0,1,2, \ldots}$ be the unique sequence of (monic) orthogonal polynomials with respect to $d \sigma$,

$$
\begin{aligned}
\pi_{k}(t) & =t^{k}+\text { lower-degree terms, } \quad k=0,1,2, \ldots, \\
\int_{-1}^{1} \pi_{n}(t) \pi_{m}(t) d \sigma & =0, \text { if } n \neq m .
\end{aligned}
$$

It is well known that every sequence of monic orthogonal polynomials satisfies a three-term recurrence relation

$$
\begin{aligned}
\pi_{k+1}(t) & =\left(t-\alpha_{k}\right) \pi_{k}(t)-\beta_{k} \pi_{k-1}(t), \quad k=0,1,2, \ldots \\
\pi_{-1}(t) & =0, \pi_{0}(t)=1
\end{aligned}
$$

with coefficients

$$
\alpha_{k}:=\alpha_{k}(d \sigma), \beta_{k}:=\beta_{k}(d \sigma)>0
$$

that are uniquely determined by the measure $d \sigma$, and by convention $\beta_{0}:=\beta_{0}(d \sigma)=$ $\int_{-1}^{1} d \sigma$. These coefficients define a tridiagonal matrix called the Jacobi matrix, with $\alpha_{k}, k=0,1, \ldots$, on the main diagonal and $\sqrt{\beta_{k}}, k=1,2, \ldots$, on the side diagonals. This matrix plays an important role for the computation of orthogonal polynomials. We can express $\pi_{n}$ as the characteristic polynomial of the $n$ th-order Jacobi matrix $J_{n}(d \sigma)=J_{n}, \pi_{n}(t)=\operatorname{det}\left(t I_{n}-J_{n}\right)$, where $I_{n}$ is the $n$th order identity matrix. The zeros of the orthogonal polynomial $\pi_{n}$ are the eigenvalues of $J_{n}$, which are also the nodes of the well-known Gauss quadrature. For a history and further extensions of these results, we refer to the extensive survey [16] of Gautschi (cf. also [42]).

We shall say that a polynomial $q_{n, r} \in \mathcal{P}_{n}$ generates a $(2 n-r-1, n, d \sigma)$ positive quadrature formula (that is, a quadrature formula which has $n$ nodes $t_{1, n}<\ldots<$ $t_{n, n}$, positive weights and $\left.\mathrm{MDE}=2 n-r-1\right)$ if all nodes $t_{1, n}, \ldots, t_{n, n}$ are zeros 
of $q_{n, r}$ and are all located in the open interval $(-1,1)$. Since MDE is $2 n-r-1$, it is easy to see that the underlying polynomial $q_{n, r}$ must be orthogonal to $\mathcal{P}_{n-r-1}$ with respect to the measure $d \sigma$. Hence, apart from a multiplicative constant, $q_{n, r}$ must be of the form

$$
q_{n, r}=\pi_{n}+\rho_{1} \pi_{n-1}+\ldots+\rho_{r} \pi_{n-r},
$$

where $\rho_{1}, \ldots, \rho_{r}$ are real constants. Such a polynomial is called a quasi-orthogonal polynomial of degree $n$ and order $r$. The quasi-orthogonal polynomials and the positive quadrature formulae have been studied by many authors. For the historical development and a number of practical computations, we refer to Askey [1], Peherstorfer [35, 36], Guessab and Rahman [22]. For an earlier paper on the subject, see Micchelli and Rivlin [31]. Recently, Xu [44] showed that a large class of quasi-orthogonal polynomials can be expressed as characteristic polynomials of a symmetric tridiagonal matrix.

Our computational method is based on the important fact that the interior nodes of (2) are zeros of a quasi-orthogonal polynomial of the form (12), which can be expressed as a characteristic polynomial of a symmetric tridiagonal matrix, that is,

$$
q_{n, r}(t)=\operatorname{det}\left(t I_{n}-J_{n, r}(d \sigma)\right),
$$

where $J_{n, r}(d \sigma)$ is defined by

$$
J_{n, r}(d \sigma)=\left[\begin{array}{ccccc}
\alpha_{0, r} & \sqrt{\beta_{1, r}} & & & 0 \\
\sqrt{\beta_{1, r}} & & \ddots & & \\
& \ddots & \ddots & \sqrt{\beta_{n-2, r}} & \\
& & \sqrt{\beta_{n-2, r}} & \alpha_{n-2, r} & \sqrt{\beta_{n-1, r}} \\
0 & & & \sqrt{\beta_{n-1, r}} & \alpha_{n-1, r}
\end{array}\right] \in \mathbb{R}^{n \times n},
$$

with

$$
\beta_{k, r}>0, k=1, \ldots, n-1, \text { and } \alpha_{k, r} \in \mathbb{R}, k=0, \ldots, n-1 .
$$

In general, such a matrix is not difficult to construct, this point being discussed further in Theorem 3.2 and Theorem 3.3. Thus, the nodes can be found numerically using one of the existing methods for computing the eigenvalues, for example, a standard QR-algorithm.

We now consider a quadrature formula of the form

$$
G_{n, r}(f)=\sum_{k=1}^{n} \lambda_{k, n} f\left(x_{k, n}\right), \lambda_{k, n} \in \mathbb{R}, x_{k, n} \in(-1,1),
$$

with $\operatorname{MDE}\left(G_{n, r}\right)=2 n-r-1$. As we mentioned before, the polynomial $q_{n, r}(t)=$ $\prod_{k=1}^{n}\left(t-x_{k, n}\right)$ is orthogonal to all polynomials of degree $\leq n-r-1$ with respect to $d \sigma$. This condition can be expressed equivalently in the form (12).

Using the symmetric tridiagonal matrix representation of quasi-orthogonal polynomials, we begin by recalling the following characterization of positive quadrature formulae, which is due to $\mathrm{Xu}$ [44, Theorem 4.1].

Theorem 3.1. Let $q_{n, r}$ generate a $(2 n-r-1, n, d \sigma)$ quadrature formula $G_{n, r}$ of the form (13). Then $G_{n, r}$ is a positive quadrature formula if and only if $q_{n, r}$ is a quasiorthogonal polynomial of degree $n$ and order $r$ that has a symmetric tridiagonal matrix representation. 
In order to present the next theorem, we have to introduce some more notation. For given linear functionals

$$
C_{l}(f)=\sum_{m=0}^{q_{l}-1} a_{l m} f^{(m)}(-1)+\sum_{p=0}^{q_{l}^{\prime}-1} b_{l p} f^{(p)}(1), \quad l=1, \ldots, k,
$$

let

$$
q=\max _{1 \leq l \leq k} q_{l}, q^{\prime}=\max _{1 \leq l \leq k} q_{l}^{\prime}, \text { and } r=q+q^{\prime}-k .
$$

We shall always assume $r \leq n, a_{l, q_{l}-1} \neq 0$ and $b_{l, q_{l}^{\prime}-1} \neq 0$ for $l=1, \ldots, k$, and we shall denote by $E_{r-1, n+q+q^{\prime}-1}$ the space defined by

$E_{r-1, n+q+q^{\prime}-1}=\left\{P \in \mathcal{P}_{n+q+q^{\prime}-1}, C_{j}(P)=P\left(x_{i, n}\right)=0, j=1, \ldots, k, i=1, \ldots, n\right\}$,

where $x_{i, n}, i=1, \ldots, n$, are the interior nodes of the quadrature formula (2). It can be easily seen that $E_{r-1, n+q+q^{\prime}-1}$ is a space of dimension $r$. Thus, there exists a set of polynomials $\left\{\Psi_{0}, \ldots, \Psi_{r-1}\right\} \subset E_{r-1, n+q+q^{\prime}-1}$ such that

$$
\Psi_{i}(t)=t^{n+k+i}+R_{i}(t), \quad i=0, \ldots, r-1,
$$

with $R_{i}$ belonging to $\mathcal{P}_{n+k-1}$ and

$$
E_{r-1, n+q+q^{\prime}-1}=\operatorname{span}\left\{\Psi_{0}, \ldots, \Psi_{r-1}\right\} .
$$

For the rest of this paper, it is convenient to consider the measure

$$
d \hat{\sigma}=(1-t)^{q^{\prime}}(1+t)^{q} d \sigma
$$

and the corresponding (monic) orthogonal polynomials

$$
\hat{\pi}_{i}(.)=\hat{\pi}_{i}(. ; d \hat{\sigma})
$$

We will also need the following quadrature formula:

$$
\hat{Q}_{n, k}(f)=\sum_{i=1}^{n} \hat{\lambda}_{i, n} f\left(x_{i, n}\right)
$$

with

$$
\hat{\lambda}_{i, n}=\left(1-x_{i, n}\right)^{q^{\prime}}\left(1+x_{i, n}\right)^{q} \lambda_{i, n}, \quad i=1, \ldots, n,
$$

where $x_{i, n}, i=1, \ldots, n$, are the nodes of the quadrature formulae $(2)$ and $\lambda_{i, n}$, $i=1, \ldots, n$, are the corresponding weights. It is easily seen that

$$
\hat{Q}_{n, k}(f)=Q_{n, k}\left((1-t)^{q^{\prime}}(1+t)^{q} f\right) .
$$

Consequently, $\hat{Q}_{n, k}$ is a $(2 n-r-1, n, d \hat{\sigma})$ positive quadrature formula. Hence, from Theorem 3.1, the polynomial that generates the quadrature formula (20) and (2) must have a symmetric tridiagonal matrix representation.

We are now ready to formulate our main theorem of this section, one that plays an important role in the subsequent development

Theorem 3.2. Suppose $q, q^{\prime}, r$ and the functions $\left\{\Psi_{0}, \ldots, \Psi_{r-1}\right\}$ are given respectively as in (14) and (16). Suppose further that $x_{1, n}, \ldots, x_{n, n}$ are $n$ points on the interval $(-1,1)$, such that $x_{i, n} \neq x_{j, n}$ for all $i \neq j$. Then the $n$ nodes $x_{1, n}, \ldots, x_{n, n}$ 
are the interior nodes of the quadrature formula (2) if and only if they are zeros of a quasi-orthogonal polynomial $q_{n, r}$ of degree $n$ and order $r$ with respect to $d \hat{\sigma}=(1-t)^{q^{\prime}}(1+t)^{q} d \sigma$, such that

$$
\int_{-1}^{1} \Psi_{i}(t) d \sigma=0, \quad i=0, \ldots, r-1 .
$$

Proof. Necessity. Assume that the nodes $x_{i, n}, i=1, \ldots, n$, are those of the quadrature formula (2). We define

$$
q_{n}(t)=\prod_{i=1}^{n}\left(t-x_{i, n}\right) .
$$

Now let $p$ be an arbitrary polynomial of degree $\leq n-r-1$; then the polynomial

$$
f(t)=(1-t)^{q^{\prime}}(1+t)^{q} q_{n}(t) p(t)
$$

is a polynomial of degree $\leq 2 n+k-1$ such that $Q_{n, k}(f)=0$. Thus, since $Q_{n, k}$ integrates exactly all polynomials of degree up to $2 n+k-1$, we have for all $p \in$ $\mathcal{P}_{n-r-1}$

$$
\begin{aligned}
\int_{-1}^{1} q_{n}(t) p(t)(1-t)^{q^{\prime}}(1+t)^{q} d \sigma & =Q_{n, k}\left((1-t)^{q^{\prime}}(1+t)^{q} q_{n}(t) p(t)\right), \\
& =0 .
\end{aligned}
$$

This means that $q_{n}$ is orthogonal to all polynomials of $\mathcal{P}_{n-r-1}$ with respect to $d \hat{\sigma}=(1-t)^{q^{\prime}}(1+t)^{q} d \sigma$. Therefore, $q_{n}$ is a quasi-orthogonal polynomial of degree $n$ and order $r$ with respect to $d \hat{\sigma}$.

For the second result, note that, since $r \leq n$, then for all $i=0, \ldots, r-1, \Psi_{i}$ is a polynomial of degree $\leq 2 n+k-1$. Then the exactness of $Q_{n, k}$ on $\mathcal{P}_{2 n+k-1}$ gives

$$
\begin{aligned}
\int_{-1}^{1} \Psi_{i}(t) d \sigma & =Q_{n, k}\left(\Psi_{i}\right), & & i=0, \ldots, r-1, \\
& =0, & & i=0, \ldots, r-1 .
\end{aligned}
$$

Thus, the necessity of the condition is proved.

Sufficiency. Assume that (22) holds and there exist $r$ real constants, $\rho_{1}, \ldots, \rho_{r}$, such that the polynomial $q_{n, r}=\hat{\pi}_{n}+\rho_{1} \hat{\pi}_{n-1}+\ldots+\rho_{r} \hat{\pi}_{n-r}$ has $n$ distinct zeros $x_{1, n}, \ldots, x_{n, n}$ on $(-1,1)$.

For a given function $f$ on $(-1,1)$, we denote by $\mathcal{I}_{n+k-1}(f ;),.\left\{h_{i, n}, l_{j, n}\right\}$ respectively the $(n+k-1)$ th degree Lagrange interpolating polynomial and the Lagrange basis with respect to the data

$$
\left\{f\left(x_{i, n}\right), \quad i=1, \ldots, n ; C_{j}(f), \quad j=1, \ldots, k\right\} .
$$

Then, we have

$$
\mathcal{I}_{n+k-1}(f ; t)=\sum_{i=1}^{n} f\left(x_{i, n}\right) h_{i, n}(t)+\sum_{j=1}^{k} C_{j}(f) l_{j, n}(t) .
$$

Since $\mathcal{I}_{n+k-1}(f ; t)=f(t)$ for all $f \in \mathcal{P}_{n+k-1}$, we obtain the following quadrature formula:

$$
\int_{-1}^{1} f(t) d \sigma=\sum_{i=1}^{n} \lambda_{i, n} f\left(x_{i, n}\right)+\sum_{j=1}^{k} \omega_{j, n} C_{j}(f)+R_{n, k}(f), \quad R_{n, k}\left(\mathcal{P}_{n+k-1}\right)=0,
$$


where

$$
\lambda_{i, n}=\int_{-1}^{1} h_{i, n}(t) d \sigma, \omega_{j, n}=\int_{-1}^{1} l_{j, n}(t) d \sigma
$$

Now, let $P$ be an arbitrary polynomial of $\mathcal{P}_{2 n+k-1}$. We define the polynomials $\Phi_{m}(t)=(1-t)^{q^{\prime}}(1+t)^{q} t^{m} q_{n, r}(t), m=0, \ldots, n-r-1$. It can be easily proved that

$$
\left\{t^{i}, i=0, \ldots, n+k-1 ; \Psi_{j}, j=0, \ldots, r-1 ; \Phi_{m}, m=0, \ldots, n-r-1\right\},
$$

form a basis for $\mathcal{P}_{2 n+k-1}$. Then $P$ can be represented uniquely in the form

$$
P(t)=\sum_{m=0}^{n-r-1} a_{m} \Phi_{m}(t)+\sum_{j=0}^{r-1} b_{j} \Psi_{j}(t)+R(t),
$$

where $R$ is a polynomial of $\mathcal{P}_{n+k-1}$. From this, it follows that

$$
\int_{-1}^{1} P(t) d \sigma=\sum_{m=0}^{n-r-1} a_{m} \int_{-1}^{1} \Phi_{m}(t) d \sigma+\sum_{j=0}^{r-1} b_{j} \int_{-1}^{1} \Psi_{j}(t) d \sigma+\int_{-1}^{1} R(t) d \sigma .
$$

For all $m=0, \ldots, n-r+1$ and $j=0, \ldots, r-1$, we have by hypothesis

$$
\int_{-1}^{1} \Phi_{m}(t) d \sigma=0 \text { and } \int_{-1}^{1} \Psi_{j}(t) d \sigma=0 .
$$

Therefore, by (23) we have

$$
\int_{-1}^{1} P(t) d \sigma=\int_{-1}^{1} R(t) d \sigma=\sum_{i=1}^{n} \lambda_{i, n} R\left(x_{i, n}\right)+\sum_{j=1}^{k} \omega_{j, n} C_{j}(R) .
$$

From (24), however, we obtain for all $i=1, \ldots, n$ and $j=1, \ldots, k$

$$
P\left(x_{i, n}\right)=R\left(x_{i, n}\right), C_{j}(P)=C_{j}(R) ;
$$

hence we have the following quadrature formula

$$
\int_{-1}^{1} P(t) d \sigma=\sum_{i=1}^{n} \lambda_{i, n} P\left(x_{i, n}\right)+\sum_{j=1}^{k} \omega_{j, n} C_{j}(P)+R_{n, k}(P), \quad R_{n, k}\left(\mathcal{P}_{2 n+k-1}\right)=0,
$$

which shows that (25) is a quadrature formula of the form (2). Therefore, by the uniqueness of the quadrature formula (2), the nodes $x_{i, n}, i=1, \ldots, n$, are indeed those of (2). This completes the proof of Theorem 3.2.

Remark 3.1. Theorem 3.2 characterizes all quadrature formulae (2). Moreover, it assures that the interior nodes of (2), which are also those of (20), are zeros of the unique quasi-orthogonal polynomial $q_{n, r}=\hat{\pi}_{n}+\rho_{1} \hat{\pi}_{n-1}+\ldots+\rho_{r} \hat{\pi}_{n-r}$, which must satisfy the orthogonality relations (22) (via the formulae (22); recall that the polynomials $\Psi_{i}$ are of the form $\Psi_{i}=q_{n, r} Q_{i}$ with $Q_{i}$ belonging to $\mathcal{P}_{q+q^{\prime}-1}$ ), and such that $q_{n, r}$ has all its zeros located in the open interval $(-1,1)$. Note also that the procedure described in Theorem 3.2 requires the evaluation of $\rho_{1}, \ldots, \rho_{r}$. The latter coefficients are a solution of a nonlinear system of $r$ equations in $r$ unknowns. In addition, as we have previously pointed out, the underlying polynomial $q_{n, r}$ must have a symmetric tridiagonal matrix representation. Hence, we can use the existing routine [18] for determining the weights and nodes in (2). These observations play a central role in the construction of (2). 
Now, we restrict our discussion by showing how quasi-orthogonal polynomials of degree $n$ and order $r=1,2,3$ or 4 can be expressed as characteristic polynomials of symmetric tridiagonal matrices. This is the content of the following theorem.

Theorem 3.3. Let $q_{n, 4}$ be a quasi-orthogonal polynomial of the form

$$
q_{n, 4}=\pi_{n}+\rho_{1} \pi_{n-1}+\ldots+\rho_{4} \pi_{n-4} .
$$

Then $q_{n, 4}$ has a symmetric tridiagonal matrix representation of the form

$$
q_{n, 4}(t)=\operatorname{det}\left(t I_{n}-J_{n}^{*}\right)
$$

with

$$
J_{n}^{*}=\left[\begin{array}{cccccc}
\alpha_{0} & \sqrt{\beta_{1}} & & & & \\
\sqrt{\beta_{1}} & \alpha_{1} & \sqrt{\beta_{2}} & & & \\
& \sqrt{\beta_{2}} & & \ddots & & \\
& & \ddots & \ddots & \sqrt{\beta_{n-2}-b_{2}} & \\
& & & \sqrt{\beta_{n-2}-b_{2}} & \alpha_{n-2}-a_{2} & \sqrt{\beta_{n-1}-b_{1}} \\
& & & & \sqrt{\beta_{n-1}-b_{1}} & \alpha_{n-1}-a_{1}
\end{array}\right]
$$

if and only if

$$
\begin{gathered}
\rho_{4}<\beta_{n-3} \beta_{n-2}, \\
b_{1} \leq \beta_{n-1},
\end{gathered}
$$

and

$$
\begin{aligned}
& b_{1}=\rho_{2}-b_{2}-a_{2}\left(a_{1}-\alpha_{n-1}+\alpha_{n-2}\right), \\
& a_{1}=\rho_{1}-a_{2}, \\
& a_{2}=\frac{\rho_{3}-b_{2}\left(\alpha_{n-3}-\alpha_{n-1}\right)-b_{2} \rho_{1}}{\beta_{n-2}-b_{2}}, \\
& b_{2}=\frac{\rho_{4}}{\beta_{n-3}} .
\end{aligned}
$$

Proof. Expanding the determinant $\operatorname{det}\left(t I_{n}-J_{n}^{*}\right)$ by its last rows, we have

$$
\begin{aligned}
\operatorname{det}\left(t I_{n}-J_{n}^{*}\right)= & \left(t-\alpha_{n-1}+a_{1}\right)\left(\left(t-\alpha_{n-2}+a_{2}\right) \pi_{n-2}(t)\right. \\
& \left.-\left(\beta_{n-2}-b_{2}\right) \pi_{n-3}(t)\right)-\left(\beta_{n-1}-b_{1}\right) \pi_{n-2}(t) \\
= & \left(t-\alpha_{n-1}+a_{1}\right)\left(\pi_{n-1}(t)+a_{2} \pi_{n-2}(t)\right. \\
& \left.+b_{2} \pi_{n-3}(t)\right)-\left(\beta_{n-1}-b_{1}\right) \pi_{n-2}(t) \\
= & \pi_{n}(t)+\left(a_{1}+a_{2}\right) \pi_{n-1}(t) \\
& +\left(b_{1}+b_{2}+a_{2}\left(a_{1}-\alpha_{n-1}+\alpha_{n-2}\right)\right) \pi_{n-2}(t) \\
& +\left(\left(a_{1}-\alpha_{n-1}+\alpha_{n-3}\right) b_{2}+a_{2} \beta_{n-2}\right) \pi_{n-3}(t) \\
& +b_{2} \beta_{n-3} \pi_{n-4}(t),
\end{aligned}
$$

where we have used the three-term relation. Since $q_{n, 4}=\operatorname{det}\left(t I_{n}-J_{n}^{*}\right)$, one readily obtains

$$
\begin{array}{ll}
a_{1}+a_{2} & =\rho_{1}, \\
b_{1}+b_{2}+a_{2}\left(a_{1}-\alpha_{n-1}+\alpha_{n-2}\right) & =\rho_{2}, \\
\left(a_{1}-\alpha_{n-1}+\alpha_{n-3}\right) b_{2}+a_{2} \beta_{n-2} & =\rho_{3}, \\
b_{2} \beta_{n-3} & =\rho_{4} .
\end{array}
$$

Solving these equations, the desired result follows. 
The main results of the previous theorem were given for the particular case $r \leq 4$. Of course, it could have been carried over to $r \geq 5$, but the problem is mainly computational. A more general form of this result has already appeared in [44, Theorem 4.1].

For the particular case $r=1\left(\rho_{2}=\rho_{3}=\rho_{4}=0\right)$, Theorem 3.3 states that every quasi-orthogonal polynomial $q_{n, 1}=\pi_{n}+\rho_{1} \pi_{n-1}$ admits a unique representation (26) with

$$
a_{1}=\rho_{1}, a_{2}=b_{1}=b_{2}=0 .
$$

This result appears in [41]. For $r=2$, the only quasi-orthogonal polynomials $q_{n, 2}=\pi_{n}+\rho_{1} \pi_{n-1}+\rho_{2} \pi_{n-2}$ which have a representation (26) with

$$
a_{1}=\rho_{1}, b_{1}=\rho_{2}, a_{2}=b_{2}=0
$$

are those that satisfy $\rho_{2} \leq \beta_{n-1}$. This characterization was proven in [31]. For $r=3$, the class of the quasi-orthogonal polynomials, which admits the matrix representation (26) under the restrictions (27) with $\rho_{4}=0$, was stated in [43] in terms of the entries corresponding to the orthonormal polynomials. Here, using the monic orthogonal polynomials as the starting point, we have easily established an explicit representation for $r \leq 4$.

If we apply Theorem 3.1 and Theorem 3.3, we arrive at the following theorem. The cases $r=1,2$ and 3 were developed in [43] and [44]. For convenience, we restated the results in terms of the recursion coefficients for the monic orthogonal polynomials.

Theorem 3.4. Let $r=1,2,3$ or 4 . Assume that the quasi-orthogonal polynomial $q_{n, r}$ is given in the form

$$
q_{n, r}=\pi_{n}+\rho_{1} \pi_{n-1}+\ldots+\rho_{r} \pi_{n-r} .
$$

Then the $(2 n-r-1, n, d \sigma)$ quadrature formula generated by $q_{n, r}$ is positive if and only if the inequalities (27) are satisfied.

As remarked previously for quadrature formulae, it is important to have all nodes in the support of the measure. In our situation, by virtue of Theorem 3.2, this is equivalent to asking when the zeros of the quasi-orthogonal polynomials are located in $(-1,1)$. One of the main difficulties in solving $(22)$ is how to select the coefficients $\left\{\rho_{k}\right\}_{k=1}^{r}$ as a solution of (22) in such a way that the corresponding quasi-orthogonal polynomial has all its zeros in $(-1,1)$. We refer the interested reader to the discussion of that point in [43] or [12]. The following theorem will be useful in the development of $\S 4$.

Theorem 3.5. Let $q_{n, 2}$ be a quasi-orthogonal polynomial of degree $n$, and order 2 , of the form

$$
q_{n, 2}=\pi_{n}+\rho_{1} \pi_{n-1}+\rho_{2} \pi_{n-2} .
$$

Suppose that $\rho_{2} \leq \beta_{n-1}$; then $q_{n, 2}$ has all its zeros in $(-1,1)$ if and only if

$$
(-1)^{n} q_{n, 2}(-1)>0 \text { and } q_{n, 2}(1)>0 .
$$




\section{Construction of the quadratures}

In this section, we first turn our attention to the numerical problem of computing a class of quadrature formulae of type (2). We will illustrate, by means of a simple example, how our characterization of (2) can be used to evaluate the free nodes as the eigenvalues of a symmetric tridiagonal matrix. We also establish that the "interior" weights are proportional to the squares of the first components of the orthonormal eigenvectors. In addition, we determine the boundary weights explicitly. Then, in the last subsection, we will compare a new class of quadrature formulae and the classical generalized Gauss-Lobatto formulae.

4.1. Gauss-Lobatto-Birkhoff type quadrature formulae. Throughout this subsection, for the sake of simplicity, we assume that $d \sigma(t)=d \sigma(-t)$ is an even measure and the support of $d \sigma$ is symmetric with respect to the origin on the interval $[-1,1]$. This restriction is only done for convenience of presentation. It will be apparent how the statement of theorems must be modified to encompass more general positive measures.

To focus our discussion, we concentrate here only on the problem of determining the nodes and weights of (2), which uses pure Neumann boundary conditions, that is, a quadrature formula of the form

$$
\int_{-1}^{1} f(t) d \sigma=Q_{n}^{G L B}(f)+R(f)
$$

where

$$
Q_{n}^{G L B}(f)=w_{0, n} f^{\prime}(-1)+\sum_{i=1}^{n} w_{i, n} f\left(x_{i, n}\right)+w_{n+1, n} f^{\prime}(1),
$$

such that $\operatorname{MDE}\left(Q_{n}^{G L B}\right)=2 n+1$. Note that (29) has the same number of evaluations of integrand and has the same MDE as the classical Gauss-Lobatto quadrature formula [16], which uses $-1,1$ and $n$ points in $(-1,1)$.

In order to show how (29) can be obtained numerically, we adopt the following notation. Let $\left\{\hat{\pi}_{n}(.)=\hat{\pi}_{n}(. ; d \hat{\sigma})\right\}_{n=0,1,2, \ldots}$ be the unique sequence of (monic) orthogonal polynomials with respect to

$$
d \hat{\sigma}:=\left(1-t^{2}\right)^{2} d \sigma
$$

and let

$$
\begin{array}{ll}
\hat{\alpha}_{k}=\hat{\alpha}_{k}(\hat{\sigma}), & 0 \leq k \leq n-1, \\
\hat{\beta}_{k}=\hat{\beta}_{k}(\hat{\sigma}), & 1 \leq k \leq n-2,
\end{array}
$$

be the recurrence coefficients for the orthogonal polynomials $\left\{\hat{\pi}_{n}(. ; \hat{\sigma})\right\}_{n=0,1,2, \ldots . .}$. Note that, in this particular case, we have (using the notations introduced in the previous section; see (14)) $k=q=q^{\prime}=2$, and $r=2$. Then it follows from (15) that

$$
E_{1, n+3}=\operatorname{span}\left\{u_{n+3}, v_{n+3}\right\},
$$

where $u_{n+3}$ and $v_{n+3}$ are two polynomials of exact degree $n+3$, which satisfy the 
interpolation conditions

$$
\begin{aligned}
& u_{n+3}\left(x_{i, n}\right)=u_{n+3}^{\prime}(-1)=u_{n+3}^{\prime}(1)=0, \\
& v_{n+3}\left(x_{i, n}\right)=v_{n+3}^{\prime}(-1)=v_{n+3}^{\prime}(1)=0,
\end{aligned}
$$

Hence, it can be easily observed that polynomials

$$
u_{n+3}(t)=\left(1-t+\frac{q_{n, 2}(1)}{q_{n, 2}^{\prime}(1)+q_{n, 2}(1)}\right)(1+t)^{2} q_{n, 2}(t)
$$

and

$$
v_{n+3}(t)=\left(1+t+\frac{q_{n, 2}(-1)}{-q_{n, 2}^{\prime}(-1)+q_{n, 2}(-1)}\right)(1-t)^{2} q_{n, 2}(t),
$$

where $q_{n, 2}(t):=\prod_{i=1}^{n}\left(t-x_{i, n}\right)$, form a basis for $E_{1, n+3}$.

Assumptions (4) and (5) are trivially true. Consequently, Theorem 2.1 assures us of the existence of a unique quadrature formula of the form (29), with respect to $d \sigma$ on the interval $[-1,1]$. This quadrature formula integrates exactly all polynomials of degree at most $2 n+1$ and such that all the nodes $x_{1, n}, \ldots, x_{n, n}$ lie in the open interval $(-1,1)$ and all the corresponding weights are positive. We also know, from Theorem 3.2, that the interior nodes of (29) are zeros of a certain quasi-orthogonal polynomial of degree $n$ and order 2 . Therefore $q_{n, 2}$ takes also the form

$$
q_{n, 2}:=\prod_{i=1}^{n}\left(t-x_{i, n}\right)=\hat{\pi}_{n}+\hat{a}_{n} \hat{\pi}_{n-1}+\hat{b}_{n} \hat{\pi}_{n-2}
$$

But, by virtue of symmetry, $q_{n, 2}$ must be even or odd for $n$ even or odd, respectively. Hence

$$
q_{n, 2}=\hat{\pi}_{n}+\hat{b}_{n} \hat{\pi}_{n-2}
$$

Again from Theorem 3.2, the polynomials $u_{n+3}$ and $v_{n+3}$ defined in (30) and (31) must satisfy the following orthogonality relations:

$$
\int_{-1}^{1} u_{n+3}(t) d \sigma=\int_{-1}^{1} v_{n+3}(t) d \sigma=0
$$

Note that $v_{n+3}$ has also the following representation:

$$
v_{n+3}(t)=\left(1+t+\frac{q_{n, 2}(1)}{q_{n, 2}^{\prime}(1)+q_{n, 2}(1)}\right)(1-t)^{2} q_{n, 2}(t) .
$$

Hence, by virtue of symmetry, the coefficient $\hat{b}_{n}$ defined in (32) must be a solution of

$$
\frac{\int_{-1}^{1}(1-t)(1+t)^{2} q_{n, 2}(t) d \sigma}{\int_{-1}^{1}(1+t)^{2} q_{n, 2}(t) d \sigma}=-\frac{q_{n, 2}(1)}{q_{n, 2}^{\prime}(1)+q_{n, 2}(1)},
$$

or equivalently, $\hat{b}_{n}$ is a solution of a quadratic equation

$$
a \rho^{2}+b \rho+c=0
$$

Then, with the help of a simple computation, we show that the solution $\hat{s}_{n}$ of (34) with the smallest modulus ensures that the polynomial

$$
q_{n, 2}=\hat{\pi}_{n}+\hat{s}_{n} \hat{\pi}_{n-2}
$$

has $n$ distinct real zeros all located in $(-1,1)$. 
Now by Theorem 3.3, the polynomial $q_{n, 2}$ admits the following matrix representation:

$$
q_{n, 2}(x)=\operatorname{det}\left(x I_{n}-J_{n}^{*}(\hat{\sigma})\right)
$$

where

$$
J_{n}^{*}(\hat{\sigma})=\left[\begin{array}{ccccc}
\hat{\alpha}_{0} & \sqrt{\hat{\beta}_{1}} & & & 0 \\
\sqrt{\hat{\beta}_{1}} & \hat{\alpha}_{1} & \ddots & & \\
& \ddots & \ddots & \sqrt{\hat{\beta}_{n-2}} & \\
& & \sqrt{\hat{\beta}_{n-2}} & \hat{\alpha}_{n-2} & \sqrt{\hat{\beta}_{n-1}-\hat{s}_{n}} \\
& & & \sqrt{\hat{\beta}_{n-1}-\hat{s}_{n}} & \hat{\alpha}_{n-1}
\end{array}\right] \in \mathbb{R}^{n \times n}
$$

Thus, the interior nodes of the quadrature formula $Q_{n}^{G L B}$ are the eigenvalues of $J_{n}^{*}(\hat{\sigma})$.

We now consider the quadrature formula based on the zeros of $q_{n, 2}$ and of the form

$$
\int_{-1}^{1}\left(1-t^{2}\right)^{2} f(t) d \sigma=\sum_{i=1}^{n}\left(1-x_{i, n}^{2}\right)^{2} w_{i, n} f\left(x_{i, n}\right)
$$

Since (29) integrates every polynomial of degree $2 n+1$ or less exactly, then (37) is valid for all polynomials of degree $\leq 2 n-3$. Now as a direct consequence of [43, Theorem 6.1], the weights $\left(1-x_{i, n}^{2}\right)^{2} w_{i, n}$ are given by

$$
\left(1-x_{i, n}^{2}\right)^{2} w_{i, n}=\frac{A_{n}}{K_{n}^{*}\left(x_{i, n}, x_{i, n}\right)} \text { for all } i=1, \ldots, n,
$$

where

$$
K_{n}^{*}(x, y)=A_{n} \sum_{k=0}^{n-1} \tilde{\pi}_{k}(x) \tilde{\pi}_{k}(y)+\tilde{\pi}_{n}(x) \tilde{\pi}_{n}(y)
$$

with $A_{n}=1-\hat{s}_{n} / \hat{\beta}_{n-1}$ and $\left\{\tilde{\pi}_{k}(.)=\tilde{\pi}_{k}(. ; \hat{\sigma})\right\}_{k=0,1,2, \ldots}$ being the set of orthonormal polynomials with respect to $d \hat{\sigma}$.

We now suppose that the eigenvectors of $J_{n}^{*}(d \hat{\sigma})$ are calculated so that

$$
J_{n}^{*}(d \hat{\sigma}) V_{i}=x_{i, n} V_{i}, \quad i=1, \ldots, n,
$$

with $V_{i}^{T} V_{i}=1$ and $V_{i}^{T}=\left(v_{1, i}, \ldots, v_{n, i}\right)$. Then, as in the ordinary Gauss quadrature formula, it follows from (38) that the Christoffel numbers $\hat{w}_{i, n}$ are expressible in terms of the first components $v_{1, i}$ of $V_{i}$ by

$$
w_{i, n}=\frac{v_{1, i}^{2}}{\left(1-x_{i, n}^{2}\right)^{2}} \beta_{0}(d \hat{\sigma}), \quad i=1, \ldots, n .
$$

We summarize this construction process in the following theorem. 
Theorem 4.1. Let $J_{n}^{*}(\hat{\sigma}), \hat{s}_{n}$ and $q_{n, 2}$ be defined as in (36) and (35). Then, for each integer $n \geq 2$ there exists a unique system of $n$ weights $w_{0, n}, \ldots, w_{n+1, n}$ such that for any polynomial $f$ in $\mathcal{P}_{2 n+1}$ we have

$$
\int_{-1}^{1} f(t) d \sigma=w_{0, n} f^{\prime}(-1)+\sum_{i=1}^{n} w_{i, n} f\left(x_{i, n}\right)+w_{n+1, n} f^{\prime}(1)
$$

where $x_{i, n}, i=1, \ldots, n$, are the $n$ distinct real eigenvalues of $J_{n}^{*}(\hat{\sigma})$,

$$
w_{n+1, n}=-w_{0, n}=\frac{\int_{-1}^{1} q_{n, 2}(t)^{2} d \sigma}{4 q_{n, 2}(1) q_{n, 2}^{\prime}(1)},
$$

and

$$
w_{i, n}=\frac{v_{1, i}^{2}}{\left(1-x_{i, n}^{2}\right)^{2}} \beta_{0}(d \hat{\sigma}), \quad i=1, \ldots, n,
$$

with $v_{1, i}$ being the first components of the normalized eigenvectors of $J_{n}^{*}(\hat{\sigma})$ corresponding to the eigenvalues $x_{i, n}$. The weights $w_{i, n}, i=1, \ldots, n+1$, are all positive and the nodes $x_{i, n}, i=1, \ldots, n$, are all in $(-1,1)$.

Proof. By virtue of the symmetry and the uniqueness of the quadrature formula (29) we have $w_{n+1, n}=-w_{0, n}$. It remains to prove (39), and the positivity of $w_{n+1, n}$. Since the quadrature formula is exact for any polynomial of degree not exceeding $2 n+1$, we easily obtain (39), and the positivity of $w_{n+1, n}$ follows immediately from the fact that

$$
q_{n, 1}^{\prime}(-1) q_{n, 1}(-1)=-q_{n, 1}^{\prime}(1) q_{n, 1}(1)
$$

and

$$
q_{n, 1}(1)>0, q_{n, 1}^{\prime}(1)=q_{n, 1}(1) \sum_{i=1}^{n} \frac{1}{1-x_{i, n}}>0 .
$$

This quadrature formula enjoys perfect symmetry; indeed we can prove easily that

$$
x_{n+1-i, n}+x_{i, n}=0, w_{n+1-i, n}=w_{i, n}, \quad i=1, \ldots, n .
$$

There are several important corollaries of Theorem 4.1, which will have applications in spectral approximations. The most important one relates to the Legendre weight function $w(t)=1$ on $[-1,1]$; the corresponding quadrature rule will be referred to as the Gauss-Lobatto-Legendre-Birkhoff type quadrature formula (GLLB). Since the following theorem is a direct consequence of the previous one, we restate the results without giving their proofs.

Theorem 4.2. Let $J_{n}$ be the nth order tridiagonal matrix defined by $\alpha_{k}$ on the main diagonal and $\sqrt{\beta_{k}}$ on the sidediagonals, where

$$
\begin{array}{ll}
\alpha_{k}=0, & k=0, \ldots, n-1, \\
\beta_{k}=\frac{k(k+4)}{(2 k+3)(2 k+5)}, & k=1, \ldots, n-2,
\end{array}
$$

and

$$
\beta_{n-1}=\frac{-\sqrt{12 n(3+n)\left(-1+3 n+n^{2}\right)}+(3+n)\left(1+2 n^{2}\right)}{(1+2 n)\left(-3+2 n+2 n^{2}\right)} .
$$


Then, for each integer $n \geq 2$, there exists a unique system of $n+2$ weights $w_{0, n}, \ldots, w_{n+1, n}$ such that for any polynomial $f$ in $\mathcal{P}_{2 n+1}$ we have

$$
\int_{-1}^{1} f(t) d t=w_{0, n} f^{\prime}(-1)+\sum_{i=1}^{n} w_{i, n} f\left(x_{i, n}\right)+w_{n+1, n} f^{\prime}(1)
$$

where $x_{i, n}, i=1, \ldots, n$, are the $n$ distinct real eigenvalues of $J_{n}$. Moreover the weights of the boundary terms are given by

$$
\begin{aligned}
w_{0, n} & =-w_{n+1, n} \\
& =\frac{24\left(-n\left(9+7 n+n^{2}\right)+(2+n){\sqrt{\Delta_{n}}}\right.}{n(1+n)(2+n)\left(3(3+n)\left(-4+n+3 n^{2}+n^{3}\right)+n(1+n){\sqrt{\Delta_{n}}}\right)},
\end{aligned}
$$

with

$$
\Delta_{n}=\sqrt{3 n(3+n)\left(-1+3 n+n^{2}\right)}
$$

and

$$
w_{i, n}=\frac{16 v_{1, i}^{2}}{15\left(1-x_{i, n}^{2}\right)^{2}}, \quad i=1, \ldots, n
$$

with $v_{1, i}$, being the first components of the normalized eigenvectors of $J_{n}$ corresponding to the eigenvalues $x_{i, n}$. The weights $w_{i, n}, i=1, \ldots, n+1$, are all positive, and the nodes $x_{i, n}, i=1, \ldots, n$, are all in $(-1,1)$.

This theorem follows from the fact that the coefficient $\hat{s}_{n}$ of the quasi-orthogonal polynomial (35) can be determined explicitly and obtained from the known relations of the Jacobi orthogonal polynomials and the following formula [2, Formula 4, p. $263]$.

$$
\int_{-1}^{1} w_{\rho, \beta}(t) p_{n}^{(\alpha, \beta)}(t) d t=\frac{2^{\beta+\rho+1} \Gamma(\rho+1) \Gamma(\beta+n+1) \Gamma(\alpha-\rho+n)}{n ! \Gamma(\alpha-\rho) \Gamma(\beta+\rho+n+2)},
$$

for $\rho<\alpha$, where

$$
w_{\alpha, \beta}(t)=(1-t)^{\alpha}(1+t)^{\beta} \quad(\alpha, \beta>-1),
$$

$p_{n}^{(\alpha, \beta)}$ being the Jacobi polynomial with parameters $\alpha, \beta$ and $\Gamma$ the gamma function. We leave the details to the reader.

The construction of quadrature formulae of type (40), in the Legendre or Chebyshev case with the Fourier-Robin boundary conditions, presents no extra difficulties; a detailed discussion on such quadrature formulae can be found in [14]. The latter are obtained by constructing analogs of Theorem 4.1 and have been implemented.

We mention also that quadrature formulae which integrate all polynomials of $\mathcal{P}_{2 n+3}$ and are of the form

$$
\int_{-1}^{1} f(t) d t=w_{0, n}(f(-1)+f(1))+\sum_{i=1}^{n} w_{i, n} f\left(x_{i, n}\right)+w_{n+1, n}\left(f^{\prime \prime}(-1)+f^{\prime \prime}(1)\right)
$$

can be obtained by the method of the present paper. Finally, the characterizations presented in this paper have been applied to the computation of a new family of quadrature formulae (2) that use end conditions common in applications. Space limitations prevent us from presenting more examples here. A detailed technical report on our numerical experiments can also be found in [14]. 
We end this section with some applications of our previous results.

4.2. Comparative numerical results. As expressed by Christoffel (cf. [16, p. $86]$ ), the use of preassigned nodes in quadrature formulae, chosen judiciously at locations where the integrand function is predominant, should be advantageous. In order to demonstrate that the use of preassigned nodes can indeed be helpful, we compare different quadrature formulae developed in this section and the classical generalized Gauss-Lobatto formulae that use the same number of evaluations of the integrand and have the same MDE. For illustration, we choose some specific functions that peak at a point in $[-1,1]$ with a severity that can be controlled by a parameter:

$$
\begin{gathered}
I_{1}(\omega)=\int_{-1}^{1} \frac{2}{1+2(\omega+t)^{2}} d t \\
=\sqrt{2}(\arctan (\sqrt{2}(1+\omega))-\arctan (\sqrt{2}(-1+\omega))), \quad \omega>0, \\
I_{2}(\omega)=\int_{-1}^{1} e^{\omega t} \sin \left(\frac{\pi}{2} t\right) d t=\frac{2 \omega \cosh t}{4 \omega^{2}+\pi^{2}}, \quad \omega>0, \\
I_{3}(\omega)=\int_{-1}^{1} e^{\omega t}\left(-3-2 t+t^{2}\right) d t=-\frac{2+4 \omega}{e^{\omega} \omega^{3}}+\frac{e^{\omega}\left(2-4 \omega^{2}\right)}{\omega^{3}}, \quad \omega>0 .
\end{gathered}
$$

Example 4.1. First we compare the approximations of $I_{1}$ obtained by using the new numerical quadrature formula GRB

$$
\int_{-1}^{1} f(t) d t=w_{0, n}^{G R B} f^{\prime}(-1)+\sum_{i=1}^{n} w_{i, n}^{G R B} f\left(x_{i, n}^{G R B}\right),
$$

and the classical Gauss-Radau GR formula

$$
\int_{-1}^{1} f(t) d t=w_{0, n}^{R} f(-1)+\sum_{i=1}^{n} w_{i, n}^{R} f\left(x_{i, n}^{R}\right) .
$$

The quadrature formula (43) is explicitly constructed in [14]. For $\omega=\omega_{m}=$ $1+3 / 2^{m}, m=1, \ldots, 11$, we determine the smallest value of $n$ such that the difference between the numerically and analytically obtained integrals is less than $10^{-15}$. The results are

$\begin{array}{cccccccccccc}m & 1 & 2 & 3 & 4 & 5 & 6 & 7 & 8 & 9 & 10 & 11 \\ G R B & 19 & 18 & 23 & 19 & 19 & 23 & 19 & 19 & 22 & 19 & 19 \\ G R & 18 & 22 & 22 & 22 & 23 & 22 & 17 & 23 & 30 & 23 & 23\end{array}$

Carefully examining these results, we observe that both quadrature formulae converge rapidly, with GRB having an edge over GR. This example also demonstrates the superiority of the new quadrature formula over GR when $\omega$ approaches 1 . Notice that the integrand $f_{\omega}(t)=\frac{2}{1+2(\omega+t)^{2}}$ exhibits a peak at $t=-\omega$.

Example 4.2. In the second example, we take the function $f_{\omega}(t)=e^{\omega t} \sin \left(\frac{\pi}{2} t\right)$, and we observe that if $\omega=0$ then $f_{0}^{\prime}( \pm 1)=0$. We therefore compare the results furnished by the quadrature formula GLLB given in Theorem 4.2,

$$
\int_{-1}^{1} f(t) d t=w_{0, n}^{G L L B} f^{\prime}(-1)+\sum_{i=1}^{n} w_{i, n}^{G L L B} f\left(x_{i, n}^{G L L B}\right)+w_{n+1, n}^{G L L B} f^{\prime}(1)
$$


with those of the classical Gauss-Lobatto quadrature formula GL,

$$
\int_{-1}^{1} f(t) d t=w_{0, n}^{G L} f(-1)+\sum_{i=1}^{n} w_{i, n}^{G L} f\left(x_{i, n}^{G L}\right)+w_{n+1, n}^{G L} f(1) .
$$

Again, for each of these quadrature formulae, we determine the smallest value of $n$ such that the difference between the numerically and analytically obtained integrals is less than $10^{-15}$. We tabulate the results for $\omega=\omega_{m}=m / 5, m=1, \ldots, 11$ :

$$
\begin{array}{cccccccccccc}
m & 1 & 2 & 3 & 4 & 5 & 6 & 7 & 8 & 9 & 10 & 11 \\
G L L B & 8 & 8 & 9 & 9 & 9 & 9 & 11 & 18 & 18 & 18 & 20 \\
G L & 9 & 9 & 9 & 15 & 15 & 15 & 19 & 19 & 19 & 26 & 18
\end{array}
$$

As we can see from the last table, the results of the proposed method are, in general, more accurate and stable than the results obtained by using the Gauss-Lobatto quadrature formula.

Example 4.3. In the following test we compare the new quadrature formula GLB1

$$
\int_{-1}^{1} f(t) d t=w_{0, n} f(-1)+\sum_{i=1}^{n} w_{i, n} f\left(x_{i, n}\right)+w_{n+1, n} f^{\prime}(1)
$$

with the classical Gauss-Lobatto quadrature formula. The quadrature formula (46) is explicitly discussed in [14]. We take the test function $f_{\omega}(t)=e^{\omega t}\left(-3-2 t+t^{2}\right)$, which satisfies $f_{\omega}(-1)=0$ and $f_{\omega}^{\prime}(1)=-4 \omega e^{\omega}$. In our next table we give the numerical results of the smallest value of $n$ for which the error is less than $10^{-15}$ for $\omega=\omega_{m}=1 / 2^{m-4}, m=1, \ldots, 9$ :

$\begin{array}{cccccccccc}m & 1 & 2 & 3 & 4 & 5 & 6 & 7 & 8 & 9 \\ G L B 1 & 5 & 5 & 10 & 10 & 18 & 3 & 6 & 12 & 24 \\ G L & 15 & 26 & 15 & 26 & 51 & 3 & 6 & 11 & 23\end{array}$

We can clearly see the superiority of the new quadrature formula.

These examples illustrate the good convergence behavior of our approach.

A natural question arises at this point concerning the quadrature error of (2). There are many quadratures that use the same number of evaluations of the integrand and have the same MDE. Then, how should one compare them? We refer to Bojanov and Nikolov [6] for other very interesting comparative general results.

\section{Applichtions to LUMPED MASS SPECTRAL APPRoXimations OF SECOND-ORDER PROBLEMS}

Several different spectral approximations have been suggested for second-order elliptic problems, and some of them have been implemented in industrial codes. The underlying idea in these methods is to approximate the unknown function by an interpolation polynomial at some preassigned (collocation) nodes. This procedure yields a system of ordinary differential equations to be solved. Most papers concerning these methods are based on collocation points that are identified with the nodes of generalized Gauss-Lobatto quadrature formulae (see [9], [37], [15], [4], among others). In certain calculations it is useful to use a set of collocation points which includes certain boundary nodes, such as the generalized Gauss-Lobatto points. This is particularly true for spectral methods, where the role of boundary conditions is even more crucial owing to their global nature. The greatest difficulty lies in finding an appropriate set of collocation points. 
In this last section, we present a new class of spectral approximations applied to second-order elliptic problems with different boundary conditions. The major difference between the proposed and the existing methods is the treatment of the boundary conditions. We will select the most accurate quadrature formula, which takes the boundary conditions into account and gives exactly a diagonal mass matrix. This property is important because our schemes can be used to introduce explicit schemes for time-dependent problems. This is the so-called lumping process. We refer the reader to [10], [37] and [24] for a review of these methods. The results reported here are only the first step in the implementation of these new methods.

In order to motivate the approach of imposing boundary conditions in the quadratures and to explain what can be achieved by this technique, we now apply the results of the foregoing sections to three important problems in the field of spectral approximations. First, we are interested in spectral discretization of the Helmholtz equation. The governing equation is

$$
\begin{cases}-\Delta u+\tau u=f & \text { on } \quad \Omega=\Lambda^{d} \\ \partial u / \partial n=0 & \text { in } \quad \partial \Omega,\end{cases}
$$

where $\Lambda=(-1,1), d=1$ or $2, \tau>0, f$ is the given data and $\partial / \partial n$ is the normal derivative operator.

5.1. One-dimensional case. The elaboration of the numerical algorithm for solving the above problem in two dimensions starts with the use of a one-dimensional case. It is for this reason that we consider the following one-dimensional case first:

$$
\left\{\begin{array}{l}
-u^{\prime \prime}+\tau u=f \\
u^{\prime}(-1)=u^{\prime}(1)=0 .
\end{array} \quad \text { on } \quad \Omega=\Lambda,\right.
$$

Several techniques are available for computing a spectral approximate solution to (48). An excellent survey of the main classes of these methods can be found in [15].

Throughout this subsection, we assume that the collocation points $x_{j}, j=$ $1, \ldots, N-1$, are the interior nodes of the quadrature formula (40) (see Theorem $4.2)$ :

$$
\int_{-1}^{1} g(t) d t=w_{0} g^{\prime}(-1)+\sum_{j=1}^{N-1} w_{j} g\left(x_{j}\right)+w_{N+1} g^{\prime}(1),
$$

which is exact over $\mathcal{P}_{2 N-1}$. A subspace of $\mathcal{P}_{N}$, important to our presentation, is defined by

$$
X_{N}^{1}=\left\{v \in \mathcal{P}_{N} ; v^{\prime}(-1)=0, v^{\prime}(1)=0\right\} .
$$

We recall that the standard pseudo spectral Legendre-Galerkin method is:

$$
\begin{aligned}
& \text { Find } u_{N} \text { in } X_{N}^{1} \text { such that } \forall v_{N} \in X_{N}^{1} \text {, } \\
& \qquad\left(\frac{d u_{N}}{d x}, \frac{d v_{N}}{d x}\right)+\tau\left(u_{N}, v_{N}\right)=\left(f, v_{N}\right),
\end{aligned}
$$

where $\left(u_{N}, v_{N}\right)=\int_{-1}^{1} u_{N} v_{N} d x$ is the standard $L^{2}$-inner product on $(-1,1)$. In order to formulate our discrete problem, we first define the discrete bilinear form 
$\langle., .\rangle_{N}^{G L L B}$ : for any functions $u$ and $v$ with continuous derivatives on $[-1,1]$, we set

$$
\langle u, v\rangle_{N}^{G L L B}=\sum_{j=1}^{N-1} w_{j} u\left(x_{j}\right) v\left(x_{j}\right)+w_{0}(u v)^{\prime}(-1)+w_{N+1}(u v)^{\prime}(1) .
$$

We always assume that the function $f \in C^{1}(\bar{\Lambda})$. We now evaluate the inner product appearing in the Legendre-Galerkin method by the quadrature formula (40) to obtain the discrete problem as:

Find $u_{N}$ in $X_{N}^{1}$ such that $\forall v_{N} \in X_{N}^{1}$,

$$
\left\langle\frac{d u_{N}}{d x}, \frac{d u_{N}}{d x}\right\rangle_{N}^{G L L B}+\tau\left\langle u_{N}, v_{N}\right\rangle_{N}^{G L L B}=\left\langle f, v_{N}\right\rangle_{N}^{G L L B} .
$$

Note that if $u_{N}$ and $v_{N}$ belong to $X_{N}^{1}$, then because of (49) the definition of $\langle., .\rangle_{N}^{G L L B}$ reduces to

$$
\begin{array}{ll}
\left\langle u_{N}, v_{N}\right\rangle_{N}^{G L L B} & =\sum_{j=1}^{N-1} w_{j} u_{N}\left(x_{j}\right) v_{N}\left(x_{j}\right), \\
\left\langle\frac{d u_{N}}{d x}, \frac{d v_{N}}{d x}\right\rangle_{N}^{G L L B} & =\sum_{j=1}^{N-1} w_{j} \frac{d u_{N}\left(x_{j}\right)}{d x} \frac{d v_{N}\left(x_{j}\right)}{d x} ;
\end{array}
$$

also note that if $u_{N}$ and $v_{N}$ belong to $X_{N}^{1}$, then we have

$$
\left\langle\frac{d u_{N}}{d x}, \frac{d v_{N}}{d x}\right\rangle_{N}^{G L L B}=\left(\frac{d u_{N}}{d x}, \frac{d v_{N}}{d x}\right) .
$$

Thus, the bilinear form $\langle., .\rangle_{N}^{G L L B}$ is a discrete scalar product in $X_{N}^{1}$. Moreover, the problem (51) is equivalent to the following variational problem:

Find $u_{N}$ in $X_{N}^{1}$ such that $\forall v_{N} \in X_{N}^{1}$

$$
\begin{aligned}
\left(\frac{d u_{N}}{d x}\right. & \left., \frac{d v_{N}}{d x}\right)+\tau \sum_{j=1}^{N-1} w_{j} u_{N}\left(x_{j}\right) v_{N}\left(x_{j}\right) \\
& =\sum_{j=1}^{N-1} w_{j} f\left(x_{j}\right) v_{N}\left(x_{j}\right)+w_{0} f^{\prime}(-1) v_{N}(-1)+w_{N+1} f^{\prime}(1) v_{N}(1) .
\end{aligned}
$$

The actual procedure of solving the above problem depends on the choice of basis functions of $X_{N}^{1}$. To this end, we take as a basis for $X_{N}^{1}$ the set $\left\{\phi_{k}\right\}_{k=1}^{N-1}$ of nodal basis functions corresponding to the degree of freedom $\left\{x_{m}\right\}_{m=1}^{N-1}$, that is given by

$$
\phi_{k} \in X_{N}^{1} \text { such that } \phi_{k}\left(x_{m}\right)= \begin{cases}1 & \text { if } k=m, \\ 0 & \text { if } k \neq m .\end{cases}
$$

Therefore, by a dimension argument, we have

$$
X_{N}^{1}=\operatorname{span}\left\{\phi_{k}, k=1, \ldots, N-1\right\},
$$

and that $\left\{\phi_{k}, k=1, \ldots, N-1\right\}$ is an orthogonal basis with respect to the discrete scalar product $\langle., .\rangle_{N}^{G L L B}$. Let us denote

$$
\begin{gathered}
\mathbf{b}_{k}=\left\langle f, \phi_{k}\right\rangle_{N}^{G L L B}=w_{0} f^{\prime}(-1) \phi_{k}(-1)+w_{k} f\left(x_{k}\right)+w_{N+1} f^{\prime}(1) \phi_{k}(1), \\
\mathbf{b}=\left(b_{1}, \ldots, b_{N-1}\right)^{T}, \\
\mathbf{U}=\left(u_{N}\left(x_{1}\right), \ldots, u_{N}\left(x_{N-1}\right)\right)^{T},
\end{gathered}
$$




$$
\begin{gathered}
a_{k, j}=\left(\frac{d \phi_{k}}{d x}, \frac{d \phi_{j}}{d x}\right), \mathbf{A}=\left(a_{k, j}\right)_{k=1, \ldots, N-1 ; j=1, \ldots, N-1}, \\
m_{k, j}=\left\{\begin{array}{ll}
w_{k} & \text { if } k=m, \\
0 & \text { if } k \neq m,
\end{array} \mathbf{M}=\left(m_{k, j}\right)_{k=1, \ldots, N-1 ; j=1, \ldots, N-1} .\right.
\end{gathered}
$$

$$
(\mathbf{A}+\tau \mathbf{M}) \mathbf{U}=\mathbf{b} .
$$

Note that the mass matrix $\mathbf{M}$ is diagonal, and for all $v_{N}$ in $X_{N}^{1}$ such that

$$
\mathbf{V}=\left(v_{N}\left(x_{1}\right), \ldots, v_{N}\left(x_{N-1}\right)\right)^{T} \neq 0 \text {, }
$$

we have

$$
\mathbf{V}^{T}(\mathbf{A}+\tau \mathbf{M}) \mathbf{V}=\left(\frac{d v_{N}}{d x}, \frac{d v_{N}}{d x}\right)+\sum_{j=1}^{N-1} w_{j} v_{N}^{2}\left(x_{j}\right)>0
$$

therefore the symmetric matrix $\mathbf{A}+\tau \mathbf{M}$ is positive definite and the discrete problem has a unique solution.

5.2. Two-dimensional case. Here $(u, v)=\int_{\Omega} u v d x d y$ is the scalar product in $L^{2}(\Omega)$. For $i, j=1, \ldots, N-1$, let us now denote $\mathbf{x}_{\mathbf{i j}}=\left(x_{i}, x_{j}\right), w_{i j}=w_{i} w_{j}$, $\phi_{i j}(x, y)=\phi_{i}(x) \phi_{i}(y)$ and

$$
X_{N}^{2}=\left\{v \in \mathcal{Q}_{N}(\Omega) ; \frac{\partial v}{\partial n}=0 \text { on } \partial \Omega\right\},
$$

where $\mathcal{Q}_{N}(\Omega)$ is the space of polynomials that are of degree less than or equal to $N$ with respect to each variable. It is obvious that

$$
X_{N}^{2}=\operatorname{span}\left\{\phi_{i j}, i, j=1, \ldots, N-1\right\} .
$$

The quadrature formula for the two-dimensional case is constructed as the tensorproduct extension of the one-dimensional case (40). From the results for the onedimensional case it is easily seen that this product quadrature formula is exact for polynomials of $\mathcal{Q}_{2 N-1}(\Omega)$. For computational reasons, it is necessary to introduce an additional bilinear form defined on $C^{1}(\bar{\Omega}) \times C^{1}(\bar{\Omega})$ by $\langle., .\rangle_{N, 2}^{G L L}$. It is constructed by replacing the integrals in the scalar product $(.,$.$) with respect to each variable$ by using the quadrature formula (40). Thus, each integral over the domain $\Omega$, in the variational form

$$
\left\{\begin{array}{l}
\text { find } u_{N} \text { in } X_{N}^{2} \text { such that } \forall v_{N} \in X_{N}^{2}, \\
\left(\nabla u_{N}, \nabla v_{N}\right)+\tau\left(u_{N}, v_{N}\right)=\left(f, v_{N}\right)
\end{array}\right.
$$

is then evaluated in the following way:

$$
\begin{array}{ll}
\left(\nabla u_{N}, \nabla v_{N}\right) & \approx\left\langle\nabla u_{N}, \nabla v_{N}\right\rangle_{N, 2}^{G L B}=\sum_{i=1, j=1}^{N-1} w_{i j} u_{N}\left(x_{i j}\right) v_{N}\left(x_{i j}\right) \\
\left(u_{N}, v_{N}\right) & \approx\left\langle u_{N}, v_{N}\right\rangle_{N, 2}^{G L L B}=\sum_{i=1, j=1}^{N-1} w_{i j} u_{N}\left(x_{i j}\right) v_{N}\left(x_{i j}\right), \\
\left(f, v_{N}\right) & \approx\left\langle f, v_{N}\right\rangle_{N, 2}^{G L B} .
\end{array}
$$

We can now pose the discrete problem as

$$
\left\{\begin{array}{l}
\text { Find } u_{N} \text { in } X_{N}^{2} \text { such that } \forall v_{N} \in X_{N}^{2} \\
\left\langle\nabla u_{N}, \nabla v_{N}\right\rangle_{N, 2}^{G L L B}+\tau\left\langle u_{N}, v_{N}\right\rangle_{N, 2}^{G L L B}=\left\langle f, v_{N}\right\rangle_{N, 2}^{G L L B}
\end{array}\right.
$$


Let us denote

$$
u_{N}(x, y)=\sum_{k, j=1}^{N-1} u_{k j} \phi_{k j}(x, y), f_{k j}=\left\langle f, \phi_{k j}\right\rangle_{N, 2}^{G L L B}
$$

and

$$
\begin{gathered}
\mathbf{U}=\left(u_{k j}\right)_{k, j=1, \ldots, N-1}, \mathbf{b}=\left(f_{k j}\right)_{k, j=1, \ldots, N-1}, \\
a_{k j l m}=\left\langle\nabla \phi_{k j}, \nabla \phi_{l m}\right)_{N, 2}^{G L L B}, \mathbf{A}=\left(a_{k j l m}\right)_{k, j, l, m=1, \ldots, N-1}, \\
m_{k j l m}=\left\{\begin{array}{l}
w_{k j} \text { if }(k, j)=(l, m), \quad \mathbf{M}=\left(m_{k j l m}\right)_{k, j, l, m=1, \ldots, N-1} . \\
0 \text { otherwise, }
\end{array}\right.
\end{gathered}
$$

Note that the mass matrix $\mathbf{M}$ is diagonal.

Taking $v_{N}(x, y)=\phi_{l m}(x, y)$ in (53) for $l, m=1, . ., N-1$, we find that (53) is equivalent to the set of algebraic equations which can be expressed in a matrix form as

$$
(\mathbf{A}+\tau \mathbf{M}) \mathbf{U}=\mathbf{b},
$$

where $\mathbf{A}+\tau \mathbf{M}$ is a symmetric positive definite matrix.

5.3. General problems. Our approach can be applied to much more complex boundary conditions, to obtain the lumping of the mass matrix results from the appropriate quadrature formulae. For the sake of simplicity, we briefly describe this method to efficiently solve the following mixed problem with Dirichlet and Neumann boundary conditions:

$$
\left\{\begin{array}{l}
-\Delta u+u=f \text { in } \Omega=\Lambda^{2} \\
u=0 \text { on } \Gamma_{i, D}, i=1,2 \\
\frac{\partial u}{\partial n}=0 \text { on } \Gamma_{i, N}, i=1,2
\end{array}\right.
$$

where

$$
\begin{aligned}
& \Gamma_{1, D}=\{(x, y) \in \Omega ; y=-1\} \\
& \Gamma_{2, D}=\{(x, y) \in \Omega ; x=-1\} \\
& \Gamma_{1, N}=\{(x, y) \in \Omega ; y=1\}, \\
& \Gamma_{2, N}=\{(x, y) \in \Omega ; x=1\} .
\end{aligned}
$$

We follow the procedure described in the pure Neumann problem, but this time we evaluate in the variational form all the integrals with respect to each variable by using the quadrature formula

$$
\int_{-1}^{1} g(t) d t=w_{0} g(-1)+\sum_{i=1}^{N-1} w_{i} g\left(x_{i}\right)+w_{n+1} g^{\prime}(1),
$$

which is exact over $\mathcal{P}_{2 N-1}$, to obtain a set of linear algebraic equations with lumped mass matrix.

Now, we turn to the specific numerical examples. In order to test our results, we have carried out numerical experiments, and some of these are illustrated by computer graphics in the following subsection. 


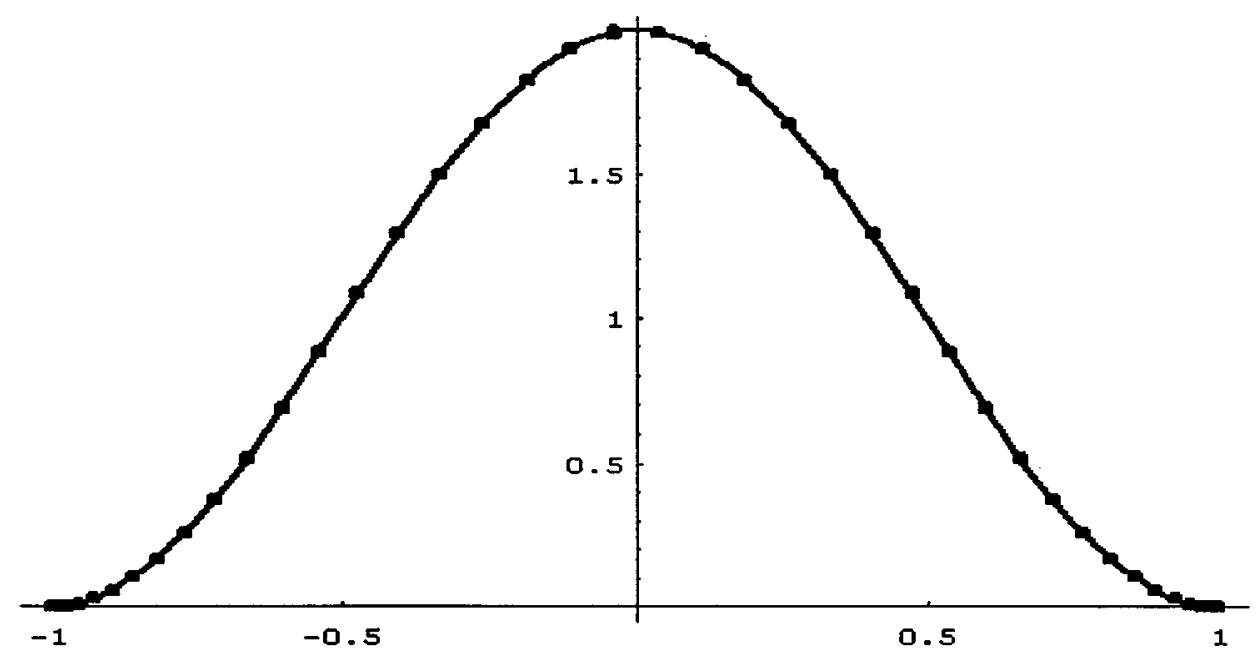

Figure 1. The computed and the exact solution of problem (56)

5.4. Implementation and numerical results. We have written FORTRAN implementations of our algorithms, using double precision arithmetic, and have applied these programs to the numerical solution of the Helmholtz equation in $\Omega=\Lambda^{d}, d=1,2$, with various boundary conditions.

We now give the descriptions of the experiments and the tables of numerical results.

\section{ONE-DIMENSIONAL TESTS}

Example 5.1. We begin with the simple 1-dimensional test problem

$$
\left\{\begin{array}{l}
-u^{\prime \prime}+u=f \\
u^{\prime}(-1)=u^{\prime}(1)=0 .
\end{array} \quad \text { on } \quad(-1,1)\right.
$$

First we take $f$ such that the exact solution is $1+\cos \pi x$. Then, we compare our approximation M1 (based on GLLB quadrature formula) with the traditional method of approximation M2 (obtained by using the classical Gauss-Lobatto-Legendre quadrature formula) and the collocation M3 method (based on the interior nodes of (40)). For various $N$ the table below contains the maximum errors at the interior nodes of the M1, M2 and M3.

\begin{tabular}{|c|c|c|c|}
\hline $\mathrm{N}$ & $\mathrm{M} 1$ & $\mathrm{M} 2$ & $\mathrm{M} 3$ \\
\hline 4 & $0.917 \mathrm{D}-02$ & $0.247 \mathrm{D}+00$ & $0.627 \mathrm{D}+00$ \\
\hline 5 & $0.196 \mathrm{D}-02$ & $0.582 \mathrm{D}-01$ & $0.125 \mathrm{D}+00$ \\
\hline 6 & $0.250 \mathrm{D}-03$ & $0.236 \mathrm{D}-01$ & $0.567 \mathrm{D}-01$ \\
\hline 7 & $0.322 \mathrm{D}-04$ & $0.236 \mathrm{D}-02$ & $0.539 \mathrm{D}-02$ \\
\hline 8 & $0.540 \mathrm{D}-05$ & $0.102 \mathrm{D}-02$ & $0.250 \mathrm{D}-02$ \\
\hline 9 & $0.432 \mathrm{D}-06$ & $0.600 \mathrm{D}-04$ & $0.141 \mathrm{D}-03$ \\
\hline 10 & $0.887 \mathrm{D}-07$ & $0.270 \mathrm{D}-04$ & $0.667 \mathrm{D}-04$ \\
\hline 11 & $0.409 \mathrm{D}-08$ & $0.104 \mathrm{D}-05$ & $0.253 \mathrm{D}-05$ \\
\hline 12 & $0.110 \mathrm{D}-08$ & $0.482 \mathrm{D}-06$ & $0.120 \mathrm{D}-05$ \\
\hline 13 & $0.204 \mathrm{D}-10$ & $0.132 \mathrm{D}-07$ & $0.328 \mathrm{D}-07$ \\
\hline
\end{tabular}


We see that the errors worsen progressively, with the errors in M1 being considerably better than in M2 and M3. We also see that the errors in M2 are close to those in M3.

For this test problem the numerical results are very promising. Using 40 grid points, the numerical results are very accurate. Figure 1 shows the computed $(\bullet)$ and exact $(-)$ solution. The reader can easily see the good agreement between computed and exact solution.

\section{TWO-DIMENSIONAL TESTS}

Example 5.2. As our second example, we take the following problem:

$$
\begin{cases}-\Delta u+u=f & \text { on } \quad \Omega=\Lambda^{2}, \\ \frac{\partial u}{\partial n}=0 & \text { in } \quad \partial \Omega,\end{cases}
$$

This time, we choose the function $f$ such that this PDE has an exact solution

$$
(1+\cos (\pi x))(1+\cos (\pi y)) .
$$

Next, using (54), the errors in the $L_{\infty}$ are given in (59), and also included in Figure 2 , the numerical solution calculated with a $12 \times 12$ grid.

\begin{tabular}{|c|c|c|c|c|}
\hline $\mathrm{N}$ & 16 & 36 & 81 & 144 \\
\hline$L_{\infty}$ & $0.183 \mathrm{E}-001$ & $0.502 \mathrm{E}-003$ & $0.864 \mathrm{E}-008$ & $0.221 \mathrm{E}-009$ \\
\hline
\end{tabular}

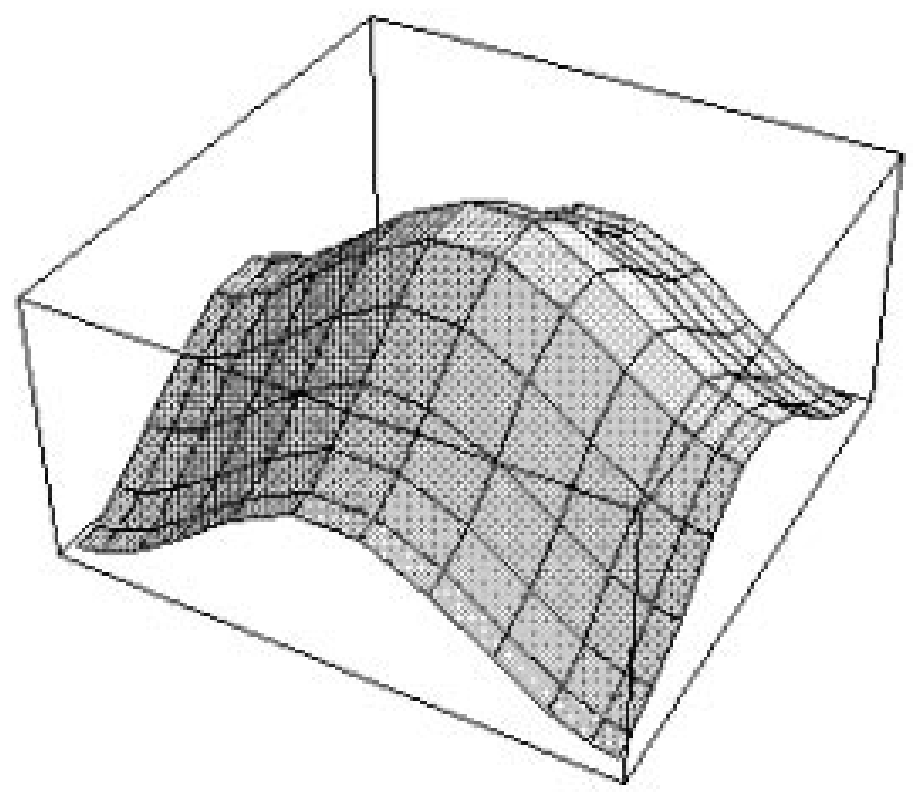

Figure 2. Computed solution of problem (58) 


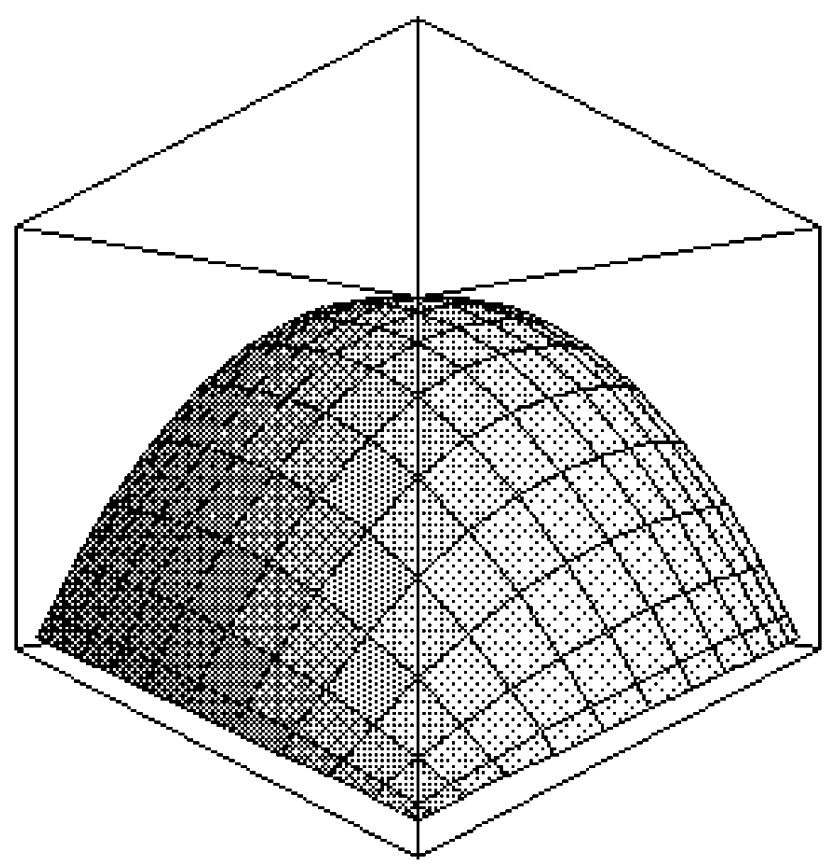

Figure 3. Computed solution of problem (60)

Our last problem involves mixed boundary conditions. The governing equation is

$$
\left\{\begin{array}{lll}
-\Delta u+u=f & \text { in } \quad \Omega=\Lambda^{2}, & \\
u=0 & \text { on } \Gamma_{i, D}, & i=1,2 \\
\frac{\partial u}{\partial n}=0 & \text { on } \Gamma_{i, N}, & i=1,2
\end{array}\right.
$$

where $\Omega$ as well as the initial data are the same as (55). Here, we choose $f$ such that this PDE has the globally smooth solution

$$
u(x, y)=(1+x)(1+y) \exp (-(x+y) / 2) .
$$

In (61), we present the maximal error at nodal points for different values of the polynomial degree, $N$. The graph of the numerical solution can be found in Figure 3 for the case of a $12 \times 12$ grid.

\begin{tabular}{|c|c|c|c|c|}
\hline $\mathrm{N}$ & 9 & 36 & 81 & 144 \\
\hline$L_{\infty}$ & $6.334 \mathrm{E}-005$ & $7.392 \mathrm{E}-007$ & $6.341 \mathrm{E}-008$ & $9.880 \mathrm{E}-009$ \\
\hline
\end{tabular}

We conclude with some comments about the quadrature formulae studied here.

In this paper, we have shown how to modify the Jacobi matrix to obtain a new class of Gaussian type quadrature formulae with prescribed evaluation of boundary values or derivatives. Our algorithm may be regarded as an extension of the classical procedure of Golub and Welsch [19] to a class of quadrature formulae with more general boundary conditions. This property makes the described quadrature formulae attractive for applications. 
The results of this paper can be improved upon and extended in several directions:

(1) The method can be extended to the construction of quadrature formulae of the form

$$
\int_{-1}^{1} f(x) d x=\sum_{i=0}^{m} a_{2 j_{i}} f^{\left(2 j_{i}\right)}(0)+\sum_{i=1}^{n} w_{i, m, n} f\left(x_{i, m, n}\right),
$$

which are exact for all polynomials in $\mathcal{P}_{2 n+2 j_{m}+1}$. Existence and uniqueness of such quadrature formulae were discussed in [8]. Note that our method can also be adapted to obtain analytically, in closed form, the boundary weights $a_{2 j_{i}}$. The interior nodes of (62) can be (and have been) computed as eigenvalues of a real symmetric tridiagonal matrix.

(2) The analysis leading to these new quadrature formulae can be extended to more general domains, for example, to semi-infinite intervals with adapted boundary conditions.

(3) The lumped mass spectral approximations have also been extended to more complicated situations, especially to fourth-order problems with more general boundary conditions. For example, if these latter are of the form $\left.u\right|_{\partial \Omega}=\left.\Delta^{2} u\right|_{\partial \Omega}$ $=0$, the idea is to use an approximate inner product to compute the mass matrix, which is based on the quadrature formula given in (42). We propose to study the theoretical and practical aspects of these problems in a future publication.

(4) In terms of a new application, we plan to extend the new algorithms to solve time-dependent problems.

The technique used in this paper can certainly be exploited in many other ways than those described here; for example, it is also useful in lumped mass finiteelement approximation, see, for instance, [9], [24] and [37]. Finally, we hope that the spectral numerical results presented here will be followed up with a theoretical analysis.

All the computations described in this paper were carried out on an IBM PC in double precision.

\section{ApPendix: Nodes AND Weights of QUADRATURE FORMUlaE}

We first show the distribution of the nodes of (40) in the interval $[-1,1]$ for $N=2, \ldots, 30$. The interlacing property and the clustering of the nodes toward the boundary are evident.

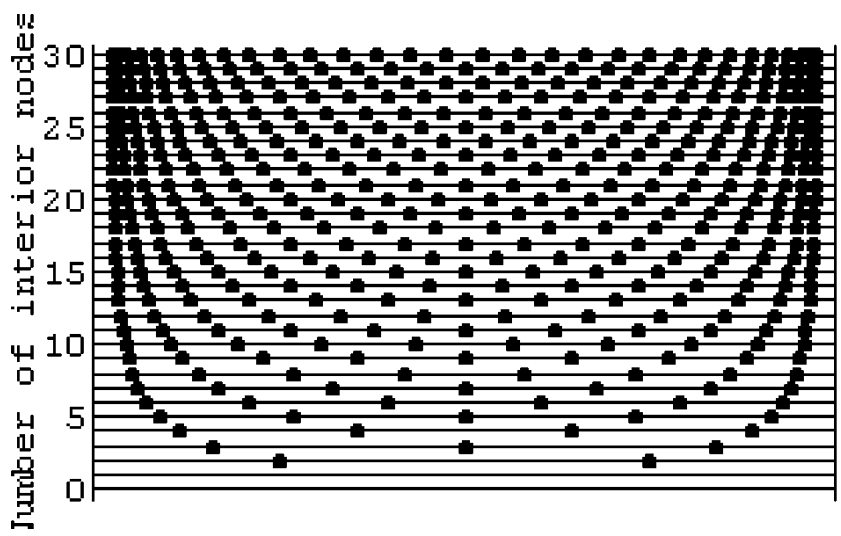


In the tables below, for $N=4(4) 16$, we give numerical values of the nodes and weights of (40) (see Theorem 4.2).

To simplify the notation for the fixed nodes $x_{0}= \pm 1$, the coefficient given in the tables corresponding to $x_{0}^{(1)}$ is the coefficient of the term $f^{\prime}\left(x_{0}\right)$ in the quadrature formulae (40).

\begin{tabular}{|c|c|}
\hline NODES & WeIGHTS \\
\hline \pm .8072338280399708 & .4180212114502938 \\
\hline \pm .2989538511730904 & .5819787885497067 \\
\hline $\pm 1.0000000000000^{(1)}$ & \pm .0044631139675894 \\
\hline NoDES & WeIGHTS \\
\hline \pm .9354789239302744 & .1424703771338546 \\
\hline \pm .7499239529233238 & .2287501679453112 \\
\hline \pm .4849763315134522 & .2964314298438723 \\
\hline \pm .1677019293183719 & .3323480250769603 \\
\hline $\pm 1.0000000000000^{(1)}$ & \pm .0004702253796003 \\
\hline NODES & WEIGHTS \\
\hline \pm .9680534976681153 & .0708606969555733 \\
\hline \pm .8741932840716901 & .1177154070361564 \\
\hline \pm .7341128563648486 & .1612389331750644 \\
\hline \pm .5545691902610785 & .1962365549042368 \\
\hline \pm .3451615188175675 & .2206900606571945 \\
\hline \pm .1171581182645735 & .2332583472717737 \\
\hline $\pm 1.0000000000000^{(1)}$ & \pm .0001135029242778 \\
\hline NoDEs & WEIGHTS \\
\hline \pm .9809891485386056 & .0422432376141590 \\
\hline \pm .9246624238272384 & .0711146068550227 \\
\hline \pm .8391511126392154 & .0994830443709286 \\
\hline \pm .7267347202244511 & .1247514431315587 \\
\hline \pm .5909799518874961 & .1460302015586128 \\
\hline \pm .4362384493353694 & .1626218477174605 \\
\hline \pm .2674795173048050 & .1739893971920475 \\
\hline \pm .0901252424115148 & .1797662215602099 \\
\hline $\pm 1.0000000000000^{(1)}$ & \pm .0000399476589447 \\
\hline
\end{tabular}

\section{ACKNOWLEDGMENT}

We thank Y. Xu of the University of Oregon for valuable discussions and careful review of the manuscript. The authors would also like to thank the referee for valuable comments.

\section{REFERENCES}

[1] R. Askey, Positive quadrature methods and positive polynomial sums, Approximation Theory 5 (C.K. Chui, L. L. Schumaker and J. D. Ward, eds.), Academic Press, New York, 1986, 1-30. MR 88j:41065

[2] P. Berckmann, Orthogonal polynomials for engineers and physicists, The Golem Press, Boulder, Colorado, 1973.

[3] C. Bernardi and Y. Maday, Some spectral approximations of one-dimensional fourth-order problems, Progress in Approximation Theory, ( P. Nevai and A. Pinkus eds.) Academic Press, San Diego (1991), 43-116. MR 92j:65176

[4] C. Bernardi and Y. Maday, Approximations spectrales de problèmes aux limites elliptiques, Spinger-Verlag France, Paris, 1992. MR 94f:65112 
[5] B. Bojanov, D. Braess and N. Dyn, Generalized Gaussian quadrature formulas, J. Approx. Theory, v. 48, 1986, pp. 335-353. MR 88c:41049

[6] B. Bojanov B. and G. Nikolov, Comparison of Birkhoff type quadrature formulae, Math. Comput., v. 54, 1990, pp. 627-648. MR 91e:65034

[7] B. Bojanov, G. Grozev and A. A. Zhensykbaev, Generalized Gaussian quadrature formulas for weak Chebychev systems, in Optimal Recovery of Functions, B. Bojanov and H. Wozniakowski (Eds.), Nova Sciences, New York, 1992, pp. 115-140.

[8] B. Bojanov and A. Guessab, Gaussian quadrature formula of Birkhoff's type, to appear.

[9] C. Canuto, M.Y. Hussaini, A. Quarteroni and T. A. Zang, Spectral methods in fluid dynamics, Springer-Verlag New York, 1988. MR 89m:76004

[10] Ph. G. Ciarlet, The finite element method for elliptic problems, North-Holland, Amsterdam, 1978. MR 58:25001

[11] Ph. J. Davis and Ph. Rabinowitz, Methods of numerical integration, Academic Press, London, 1984. MR 86d:65004

[12] E. A. Van Doorn, Representation and bounds for zeros of orthogonal polynomials and eigenvalues of sign-symmetric tri-diagonal matrices, J. Approx. Theory, v. 51, 1987, pp. 254-266. MR 88j:33018

[13] A. Ezzirani, Construction de formules de quadrature pour des systèmes de Chebychev avec applications aux méthodes spectrales, Thèse de l'Université de Pau, France, 1996.

[14] A. Ezzirani and A. Guessab, A fast algorithm for Gaussian type quadrature formulae with mixed boundary conditions, and some lumped mass spectral approximations, U.A. CNRS 1204. 6(1998).

[15] D. Funaro, Polynomial approximation of differential equations, Spinger-Verlag, Berlin, 1992. MR 94c:65078

[16] W. Gautschi, A survey of Gauss-Christoffel quadrature formulae, in E. B. Christoffel (P. L. Butzer and F. Fehér, Eds.), Birkhäuser, Basel, 1981, pp. 72-147. MR 83g:41031

[17] W. Gautschi and S. Li, Gauss-Radau and Gauss-Lobatto quadrature with double end points, J. Comput. Appl. Math., v. 34, 1991, pp. 343-360. MR 92c:65032

[18] W. Gautschi, Algorithm 726: ORTHPOL - A package of routines for generating orthogonal polynomials and Gauss-type quadrature rules, ACM Trans. Math. Software 20(1994), 21-62.

[19] G. H. Golub and J. H. Welsch, Calculation of Gauss quadrature rules, Math. Comp., v. 23, 1969, pp. 221-230. MR 39:6513

[20] G. H. Golub, Some modified matrix eigenvalue problems, SIAM Rev., v. 15, 2(1973), pp. 318-334. MR 48:7569

[21] G. H. Golub and J. Kautsky, Calculation of Gauss quadratures with multiple free and fixed knots, Numer. Math., v. 41, 1983, pp. 147-162. MR 84i:65030

[22] A. Guessab and Q. I. Rahman, Quadrature formulae and polynomial inequalities, J. Approx. Theory, v. 90, 1997, pp. 255-282. CMP 97:16

[23] A. Guessab and G. V. Milovanović, An algorithm for Gauss-Birkhoff type quadrature formulae, to appear.

[24] T. Hughes, The finite element method: linear static and dynamic finite element analysis, Prentice-Hall, 1987. MR 90i:65001

[25] K. Jetter, A new class of Gaussian quadrature formulae based on Birkhoff type data, SIAM J. Numer. Anal., v. 5, 1982, 1080-1089. MR 84d:41054

[26] S. Karlin and W. J. Studden, Tchebycheff systems: with applications in analysis and statistics, Wiley, 1966. MR 34:4757

[27] M. G. Krein, The ideas of P. L. Chebyshev and A. A. Markov in the theory of limiting values of integrals and their further developments, Uspekhi Mat. Nauk, v. 6, 1951, no. 4, 3-120 (Russian); Amer. Math. Soc. Transl. Ser. 2, 12 (1959), pp. 1-122. MR 13:445c; MR 22:3947a

[28] G. G. Lorentz, K. Jetter and S. D. Riemenschneider, Birkhoff interpolation, Encyclopedia of Mathematics and its Applications, 19, Addison-Wesley, Reading, Mass., 1983. MR 84g:41002

[29] A. A. Melkman, Interpolation by splines satisfying mixed boundary conditions, Israel J. Math.,19 (1974), pp. 369-381. MR 51:8683

[30] C. A. Micchelli and A. Pinkus, Moment theory for weak Chebyshev systems with applications to monosplines, quadrature formulae and best one-sided $\mathrm{L}^{1}$-approximation by spline functions with fixed knots, SIAM J. Math. Anal. 8 (1977), pp. 206-230. MR 55:8627 
[31] C. A. Micchelli and T. J. Rivlin, Numerical integration rules near Gaussian formulas, Israel J. Math., v. 16, 1973, pp. 267-299. MR 51:2255

[32] C. A. Micchelli and T. J. Rivlin, Quadrature formulae and Hermite-Birkhoff interpolation, Advances in Mathematics, v. 11, 1973, pp. 93-112. MR 47:7289

[33] G. V. Milovanović, Construction of s-orthogonal polynomials and Turán quadratures, in Approx. Theory III, Niš, 1987, (ed. G. V. Milovanović) Univ. Niš, 1988, pp. 311-328. MR 89g:65023

[34] B. N. Parlett, The symmetric eigenvalue problem, Prentice-Hall, Englewood Cliffs, 1980. MR 81j:65063

[35] F. Peherstorfer, Characterization of positive quadrature formulae, SIAM J. Math. Anal., v. 12, 1981, pp. 935-942. MR 82m:65021

[36] F. Peherstorfer, Characterization of positive quadrature formulae II, SIAM J. Math. Anal. v. 15, 1984, pp. 1021-1030. MR 86a:65025

[37] A. Quarteroni and A. Valli, Numerical approximation of partial differential equations, Spinger-Verlag, Berlin, (1994). MR 95i:65005

[38] T. J. Rivlin, "Chebyshev polynomials: From approximation theory to algebra and number theory," 2nd ed., Wiley, New York, 1990. MR 92a:41016

[39] H. J. Schmid, A note on positive quadrature rules, Rocky Mountain J. Math., v. 19, 1989, pp. 395-404. MR 90k:41041

[40] D. D. Stancu, Sur quelques formules générales de quadrature du type Gauss-Christoffel, Mat. (Cluj), v. 1, 1959, pp. 167-182. MR 22:11078

[41] G. Szegö, Orthogonal Polynomials, Colloquium Publication, v. 23, 4th ed., Amer. Math. Soc., Providence, R. I., 1975. MR 51:8724

[42] D. S. Watkins, Some perspectives on the eigenvalue problem, SIAM Review, v. 35, 1993, pp. 430-471. MR 94h:65039

[43] Y. Xu, Quasi-orthogonal polynomials, quadrature, and interpolation, J. Math. Anal. Appl., v. 182, 1994, pp. 779-799. MR 95a:42035

[44] Y. Xu, A characterization of positive quadrature formulae, Math. Comp., 62, 1994, pp. 703718. MR 94h: 41067

Laboratoire de Mathématiques Appliquées, UpRes A 5033, Associé au CNRS, UniVersité de Pau, 64000, France

E-mail address: guessab@univ-pau.fr 